\title{
APORTACIONES AL CATÁLOGO FLORÍSTICO DE LA PROVINCIA DE LEÓN. II
}

\author{
Carbó Nadal, R.*, Mayor López, M.**, \\ Andrés Rodríguez, J.* \& Losa Quintana, J. M. ${ }^{a *}$
}

\section{RESUMEN}

Se enumeran 512 especies vegetales de la provincia de León (España) recogidas en 693 localidades, se hacen breves anotaciones ecológicas de las mismas, así como de su distribución y comportamiento fitosociológico. Se realiza, al mismo tiempo, la revisión bibliográfica a nivel provincial.

\section{SUMMARY}

We note 512 vegetable specia in the province of León (Spain), collected in 693 places and we make brief ecologic remarks about them, and also about their distribution and phytosocilogical behaviour. We made, at the same time, the bibliographical review at provincial level.

\section{CATÁLOGO FLORISTICO Y REVISIÓN BIBLIOGRÁFICA PROVINCIAL}

\section{EQUISETACEAE}

\section{Equisetum sylvaticum L.}

Puebla de Lillo (17-6-73), bajo Pinus silvestris L.

Circumboreal.

\section{ADIANTACEAE}

\section{Adiantum capillus-veneris $\mathrm{L}$.}

Puente de Domingo Flórez (6-5-72), en paredones rocosos. Inmediaciones de la ciudad de León (COLMEIRO).

Pseudomediterránea-Atlántica.

(*) Departamento de Botánica. Facultad de Ciencias (León).

(**) Departamento de Botánica. Facultad de Ciencias. Universidad de Oviedo.

$\left({ }^{\star * *}\right.$ Departamento de Botánica. Facultad de Ciencias (León). 


\section{GYMNOGRAMMACEAE}

Anogramma leptophylla (L.) Link

Gymnogramma leptophylla (L.) Desv.

Puente de Domingo Flórez (19-5-72), en dolomías. Villafranca del Bierzo (LANGE).

Cosmopolita.

\section{ASPLENIACEAE}

\section{Asplenium trichomanes $\mathrm{L}$.}

subsp. quadrivalens D. E. Meyer

Castillo de Cornatel (19-5-72), en dolomías. Puerto Ventana (RIVAS-MARTINEZ y col.).

Asplenietea rupestris.

\section{ASPIDIACEAE}

Dryopteris carthusiana (Villar) H. P. Fuchs

D. spinulosa Watt.

Alto de Leitariegos (13-6-71).

Eurosiberiana. Fagion.

Polystichum setiferum (Forskal) Woynar

Aspidium aculeatum $\mathrm{Sw}$.

Alto de Leitariegos (13-6-71), en brezal. Pico de Arvas (DURIEU). Riaño (LOSA).

Subcosmopolita. Euro-mediterránea y atlántica. Fagitalia.

Polypodium australe Fée

Castillo de Cornatel (14-4-72), en rocas dolomíticas.

Subcosmopolita. Asplenietea rupestris.

\section{Polypodium vulgare $\mathrm{L}$.}

Castillo de Cornatel (14-4-72), en rocas dolomíticas. San Pedro de Montes (POURRET). Villafranca del Bierzo (LANGE). Picos de Europa (LASCOMBES). Riaño (LOSA).

Subcosmopolita.

\section{CUPRESSACEAE}

Juniperus communis $\mathrm{L}$.

subsp. communis

Citado profusamente en la provincia. El Castrillino (1-8-69), formando bosquetes. Europea.

\section{SALICACEAE}

\section{Salix caprea L.}

Pantano de Bárcera (12-5-73). Citado en el centro de la provincia y en el Bierzo. Euroasiática. 


\section{BETULACEAE}

Betula pubescens Ehrh.

subsp. celtibérica (Rothm./Vasc.) Rivas Martínez.

Pinar de Puebla de Lillo (12-6-73). Numerosas citas en la zona montañosa.

Euroasiática. Betulo-Adenostyletea.

\section{FAGACEAE}

Quercus petraea (Mattuschka) Liebl.

Q. sessiliflora Salisb.

Puebla de Lillo (20-10-73). Numerosas citas en el nordeste de la provincia.

Atlántica. Quercion robori-petraeae.

Quercus robur $\mathrm{L}$.

Cerecedo, Boñar (22-6-74). Substrato silíceo. Muy citado en La Montaña.

Europea. Quercion robori-petreae.

Quercus faginea Lamk.

subsp. faginea

Quercus valentina Cav.

Crémenes (12-6-72).

Ibérica. Quercion valentinae.

\section{ULMACEAE}

\section{Ulmus minor Miller}

$U$. carpinifolia G. Suckow; U. campestris auct., non L.

$U$. foliacea Hayek; U. glabra Miller, non Hudson

Villafranca del Bierzo (14-4-72). No muy numerosas citas, y muy diseminadas en la provincia.

Paleotemplada.

\section{CANNABACEAE}

\section{Humulus lupulus $\mathrm{L}$.}

Toral de los Vados (14-4-72). Moral del Condado (8-7-69). Siempre nemoral. Villafranca del Bierzo (LANGE).

Euroasiática.

\section{MORACEAE}

Ficus carica L.

Puente de Domingo Flórez (5-5-72). Sobre sustrato dolomítico. Santa Marina del Sil y Pardamaza (I.N.I.A.).

Mediterránea. 


\section{SANTALACEAE}

\section{Osyris alba L.}

Villafranca del Bierzo (14-4-72). Puente de Domingo Flórez (23-4-72). San Juan de Paluezas (12-5-73). Villafranca del Bierzo (LANGE). Cacabelos (COLMEIRO). Circunmediterránea. Quercetalia ilicis.

Thesium humifusum DC.

Benllera (29-6-70), en brezales.

Subatlántica.

\section{POLYGONACEAE}

Bilderdykia convolvulus (L.) Dumort.

Polygonum convolvulus L.

Valporquero (19-8-72), en bordes de campos de cultivo de cereales. Villafranca del Bierzo (LANGE). Riaño (LOSA). Portilla de la Reina (I.N.I.A.).

Circumboreal. Secalinion.

\section{Polygonum viviparum $\mathrm{L}$.}

Vega de Liordes, Picos de Europa (30-7-73). Mampodre (LOSA). Picos Albos y Peña de Orniz (LAINZ). Cordiñanes y Maraña (I.N.I.A.).

Orófila alpina.

\section{Rumex patientia L.}

Lorenzana (10-6-73), en borde de camino. También citado en La Montaña y Bierzo. Europea. Chenopodietalia.

Rumex suffruticosus Gay

Peña Cuiña, Ancares (25-7-70). Citado también en La Montaña.

Endemismo ibérico. Androsacetalia alpinae.

\section{PORTULACACEAE}

Montia fontana L.

subsp. fontana

Montia minor Gmel.

Villafranca del Bierzo (14-4-72).

Subcosmopolita.

Montia fontana $\mathrm{L}$.

subsp. chondrosperma (Fenzl.) Walters.

Montia rivularis $\mathrm{Gmel}$.

Val de San Lorenzo (14-5-74), formando grandes madejas en una fuente. Nocedo (BORJA). Vegamián (I.N.I.A.).

Europea. Cardamineto-Montion.

\section{CARYOPHYLLACEAE}

Arenaria aggregata (L.) Loisel

subsp. querioides Pourr.

Valporquero (19-8-72), en pedregales calcáreos. Algunas citas bibliográficas en la Meseta y zonas montañosas de la provincia.

Mediterránea. 
Minuaríia rubra (Scop.) Mc Neill

Alsine fasciculata M. K.

Lodares (29-6-73), sobre calizas. Cuenca del río Luna (LAfNZ).

Submediterránea. Orofilo-alpina.

Minuartia villarsi (Balbis) Wilzeck/Chenevard.

Valporquero (19-8-72). Puebla de Lillo (1-7-73). En fisuras de rocas calizas. Citado profusamente en La Montaña.

Orófila alpina. Festucion burnatii.

Stellaria alsine Grimm

Stellaria uliginosa Murray

Lodares, Pantano del Porma (29-6-72), en zonas muy húmedas sobre sustrato cuarcítico. Citada en La Montaña y El Bierzo.

Circumboreal. Montio-Cardaminetalia.

Cerastium dichotomum L.

Ardoncino (27-4-74), en cultivos arcillosos.

Mediterráneo y Asia.

Moenchia erecta (L.) Gaertner, B. Meyer/Schreb.

Cerastium erectum (L.) Cosson/Germ.

Llamas de Rueda (9-5-71). Puerto de Manzanal (23-5-71). Lillo del Bierzo (6-6-71). Rodanillo (17-6-71). En pastizales ácidos. Mitad norte de la provincia y en El Bierzo. Mediterránea. Moenchion erectae.

Sagina apetala Ard.

Colegiata de Arbas (9-6-73). Riaño (LOSA). León (TUXEN/OBERDORFER).

Mediterránea.

Sagina procumbens $\mathrm{L}$.

subsp. muscosa (Jordan) Nyman

Sagina fasciculata Boiss.

S. pyrenaica Rouy

Puerto de San Glorio (6-8-72). Puerto del Pontón (LOSA/MONTSERRAT). Burón, Oseja de Sajambre (I.N.I.A.).

Pirineos y Cordillera cantábrica. Salicetea herbaceae.

Sagina procumbens $\mathrm{L}$.

subsp. procumbens

Pinar de Puebla de Lillo (16-6-73), en zonas muy pisoteadas. Villafranca del Bierzo (LANGE). Entre Crémenes y Las Salas (LOSA/MONTSERRAT). Isoba, Burón y Portilla de la Reina (I.N.I.A.).

Subcosmopolita. Nardion.

Paronychya polygonifolia (Vill.) DC.

Peña Cuiña, Ancares (25-7-70). Catoute (26-7-73). Siempre sobre sustrato silíceo. Mampodre (LOSA). Colegiata de Arbas (GANDOGER). Cordiñanes (I.N.I.A.).

Montañas del sur de Europa. Seneción leucophyllae. 


\section{Herniaria glabra $\mathrm{L}$.}

Virgen del Camino (12-5-73). Numerosas citas en La Montaña y El Bierzo.

Paleotemplada. En Hordeion leporini.

Spergularia capillacea (Kindb./Lange) Willk.

Peña Cuiña, Ancares (25-7-70). Catoute (26-7-73). En zonas muy pisoteadas. Riaño (LÁINZ). Puerto del Manzanal (LANGE).

Endemismo ibérico.

Spergularia segetalis (L.) G. Don fil.

Llamas de Rueda (9-5-71). Herreros de Jamuz (20-5-71).

Mediterránea-Subatlántica.

Lychnis flos-cuculi $\mathrm{L}$.

Puente Orugo (30-6-71). Armada (22-6-74). Numerosas citas para La Montaña y El Bierzo. Siempre en prados de siega muy húmedos o encharcados.

Eurosiberiana. Molinietalia.

\section{Petrocoptis grandiflora Rothm.}

Puente de Domingo Flórez (14-4-72), en rocas dolomíticas cubriendo superficies casi verticales. También citada en El Bierzo.

Endemismo regional. Saxifragion trifurcato-canaliculatae.

Petrocoptis viscosa Rothm.

Castillo de Cornatel (14-4-72), en dolomías. También citada en El Bierzo.

Endemismo regional. Saxifragion trifurcato-canaliculatae.

Silene alba (Miller) E. H. L. Krause

subsp. divaricata (Reichenb.) Walters

Toral de los Vados (14-4-72). Pantano de Bárcena (12-5-73). Pola de Gordón (315-73). Salentinos (26-7-73).

Mediterránea.

\section{Silene ciliata Pourret}

Catoute (26-7-73). También citada en La Montaña.

Europa occidental.

Silene colorata Poiret

Puente Villarente (22-10-72).

Mediterránea.

Silene conica $\mathrm{L}$.

subsp. cónica

Virgen del Camino (12-5-73).

Paleotemplada.

\section{Silene gallica $\mathrm{L}$.}

Herreros de Jamuz (3-6-73), en bordes de cultivos. Montañas de León (LANGE). Subcosmopolita. 
Silene mellifera Boiss./Reuter

Vegacervera (17-7-70), sobre sustrato calcáreo.

Endemismo ibérico.

Silene portensis L.

Puente de Vilarente (22-10-72), en cultivos en barbecho.

Mediterránea-Atlántica.

Silene psammitis Link

Herreros de Jamuz (28-5-73), en herbazales y campos de cultivo.

Endemismo ibérico.

Silene saxifraga $\mathrm{L}$.

Puente de Domingo Flórez (23-5-72), en rocas dolomíticas. También citada en La Montaña y El Bierzo.

Euroasiática. Thlaspeetea.

Vaccaria pyramidata Medicus

V. segetalis Garcke; Saponaria vaccaria L.

Valdelafuente (30-6-74), en cultivos de cereales en barbecho. Riaño y Oseja de Sajambre (I.N.I.A.).

Mediterránea. Secalinetalia.

Dianthus armeria L.

Armada, Pantano del Porma (9-7-72). Villafranca del Bierzo (LANGE). Riaño (LOSA). Primajas y Abelgas (I.N.I.A.).

Europea.

Dianthus langeanus Willk.

D. hispanicus Asso. var. occidentalis Willk.

Peña Cuiña, Ancares (25-7-72). Catoute (26-7-73). Citas bibliográficas de La Montaña y El Bierzo, no muy numerosas.

Noroeste de la Península.

Dianthus monspessulanus $\mathrm{L}$.

subsp. monspesulanus

Puebla de Lillo (26-7-69). Puente de Domingo Flórez (19-5-72). Cofiñal (13-8-72). Valporquero (19-8-72). Siempre en pastizales. Muy citado en toda la zona montañosa de la provincia.

Mediterránea-Atlántica.

Dianthus subacaulis Vill.

subsp. brachyanthus (Boiss.) P. Fourn.

Puerto de Piedrafita, Cármenes (11-7-70). Lodares (29-6-72). Vega de Gordón (315-73). Camposolillo (29-6-73).

Orófila europea. Elyno-Seslerietea. 


\section{CERATOPHYLLACEAE}

\section{Ceratophyllum demersum $\mathrm{L}$.}

Valencia de D. Juan (10-5-72), en una acequia.

Subcosmopolita.

\section{RANUNCULACEAE}

\section{Helleborus viridis $\mathrm{L}$.}

subsp. cocidentalis (Reuter) Schyffner.

Vegarada (18-7-70). Puebla de Lillo (8-6-74). Muy citado en La Montaña y El Bierzo. Mediterránea-Atlántica. Fagion.

\section{Anemone palmata $\mathrm{L}$.}

Herreros de Jamuz (6-5-73), en pastizal.

Mediterránea occidental.

Ranunculus abnormis Cutanda/Willk.

Santa Colomba de Somoza (11-5-74). En prados de siega sobre sustrato silíceo. Ibérica.

\section{Ranunculus aquatilis $\mathrm{L}$.}

Val de San Lorenzo (18-3-73). San Miguel del Camino (12-5-73). En aguas con poco movimiento.

Cosmopolita.

\section{Ranunculus auricomus $\mathrm{L}$.}

Puerto de San Isidro (8-6-74). Pantano del Porma (30-6-74). Busdongo (LAfNZ). Europea.

\section{Ranunculus trichophyllus Chaix}

Ranunculus divaricatus Schrank.

Lago de Carucedo (23-4-72). Maraña (LOSA). Pto. de Pinos (LAínZ).

Europea.

\section{Ranunculus fluitans Lam.}

Puebla de Lillo, Fuentelavilla (16-6-73).

Circumboreal.

\section{Ranunculus gouanii Willd.}

Puerto de San Isidro, Cebolledo (2-7-73), sobre sustrato calcáreo. Puerto de Tarna y Coriscao (LAf́nZ). Picos de Europa (LASCOMBES).

Francia y España. Festucion burnatii.

\section{Ranunculus gregarius Brot.}

$R$. nevadensis Willk. $R$. escurialensis Boiss./Reuter. $R$. carpetanus Boiss./Reuter. Villargusán (26-6-69). Los Bayos, Puerto de la Magdalena (17-6-71). Puente de Domingo Flórez (23-4-72). Pantano de Bárcena (12-5-73). Vega de Gordón (31-5-73). Boca de Huérgano (I.N.I.A.).

Ibérica. 


\section{Ranunculus hederaceus L.}

Torrestío (10-6-73), en acequias. Villafranca del Bierzo (LANGE). Montañas de León (CAMMARA NIÑO).

Atlántica. Potamion.

Ranunculus ololeucos Lloyd

Valcabado del Páramo (11-5-72). Turberas bajo el Lago Ausente, en Puebla de Lillo (ALLORGE).

Europa occidental.

\section{Ranunculus parviflorus L.}

De Villafranca a Toral de los Vados (14-4-72). Valcabado del Páramo (12-5-72). Ribaseca (28-5-73). San Pedro de Montes (POURRET). Villafranca del Bierzo (LANGE).

Mediterránea-Atlántica.

Ranunculus pseudofluitans (Syme) Newbould

Valcabado del Páramo (14-5-72). Toralino de la Vega (14-5-72), en charcas.

Europea. En Isoeto-Nanojuncetea.

\section{Ranunculus sardous Crantz}

Lago de Carucedo (23-4-72). Valcabado del Páramo (12-5-72). Mirantes de Luna (10-6-73). En zonas muy húmedas, casi encharcadas.

Europea. Isoeto-Nanojuncetea.

Myosurus minimus $\mathrm{L}$.

Valcabado del Páramo (12-5-72). En charcas.

Circumboreal. Isoeto-Nanojuncetea.

\section{Thalictrum flavum $\mathrm{L}$.}

Barrio de Nuestra Señora (18-6-73). La Bañeza (LANGE). León (GANDOGER).

Euroasiática.

Thalictrum minus $\mathrm{L}$.

La Uña (13-8-72). Puebla de Lillo, Cebolledo (8-7-73). San Pedro de Montes (POURRET). Riaño (LOSA). Reyero (I.N.I.A.).

Euroasiática. Populion albae.

\section{PAEONIACEAE}

Paeonia officinalis $\mathrm{L}$.

subsp. humilis (Retz.) Cullen/Heywood

Suroeste de Europa.

Valporquero (19-8-73). Los Apóstoles, sobre San Adrián de Valdueza (LAfNZ).

\section{PAPAVERACEAE}

\section{Papaver dubium $\mathrm{L}$.}

Villasabariego (11-6-72), impurificando cultivos de cereales. Muy abundante en la provincia.

Mediterránea. Secaletalia. 


\section{Papaver hybridum L.}

P. hispidum Lam.

Citas bibliográficas no muy abundantes, pero bastante diseminadas en la provincia.

Villasabariego (11-6-72), impurificando campos de cultivo.

Circunmediterránea. Secaletalia.

Papaver somniferum L.

Quintana de Rueda (6-7-76), en escombros cerca de la carretera. Citado esporádicamente en la provincia.

Cultivada en el centro y oeste de la región mediterránea.

Hypecoum procumbens $\mathrm{L}$.

Vegas del Condado (6-5-73). Valencia de D. Juan (4-5-74). En cultivos. Montes de León (LANGE).

Mediterránea y de Asia. Secalinion.

Corydalis bulbosa (L.) DC.

subsp. bulbosa

Puerto de San Isidro, Cebolledo (16-6-73). Millaró y San Isidro (LAfNZ).

Europea. Querco-Fagetea.

Fumaria martinii Clavaud

Castrillo de los Polvazares (2-5-74). En campos de cultivo. No encontramos ninguna cita de esta especie en los trabajos consultados.

Ibérica.

Fumaria muralis Sond.

Puente de Domingo Flórez (23-4-72).

Atlántica.

Fumaria parviflora Lam.

Villasabariego (4-5-73), en campos de cultivo. Montañas de León (LANGE).

Mediterránea. Chenopodietalia.

\section{CRUCIFERAE}

Sisymbrium austriacum Jacq.

subsp. austriacum

Puerto de San Glorio (6-8-72). Herreros de Jamuz (4-8-72). Viaducto de Aralla (10-6-73). Vega de Liordes (30-7-73). Riaño (LOSA).

Europa central y occidental.

Sisymbrum austriacum Jacq.

subsp. chrysanthum (Jordan) Rouy/Fouc.

Valporquero (19-8-72). Riaño (LOSA).

Pirineos y Cordillera Cantábrica.

Sisymbrium irio L.

Valencia de D. Juan (20-5-73), en zonas con abundante materia orgánica. Subcosmopolita. 
Sisymbrium officinale (L.) Scop.

Jiménez de Jamuz (29-4-73), en bordes de cultivos. San Pedro de Montes (POURRET).

Cosmopolita. Hordeion murinii.

Descurainia sophia (L.) Webb

Sisymbrium sophia L.

Valencia de D. Juan (2-5-73). Borde de carretera. La Bañeza (LANGE).

Subcosmopolita. De Chenopodio-Stellarietea que se introduce en Secalinion.

Murbeckiella boryi (Boiss.) Rothm.

Braya pinnatifida Willk.

Catoute (26-7-73). Sustrato silíceo. Circo glaciar de Cubil del Can (LOSA/

MONTSERRAT).

Orófila alpina y pirenaica.

Hugueninia tanacetifolia (L.) Reichenb.

subsp. sufruticosa (Coste/Soulie) P. W. Ball.

Valporquero (19-8-72). Algunas citas, no muy numerosas, para La Montaña.

Orófila alpina. Alneto-Adenostylion.

Arabidopsis thaliana (L.) Heynh.

Arabis thaliana L. Sisymbrium thalianum (L.) Gay

Llamas de Rueda (9-5-71). Toral de los Vados (14-4-72). Crémenes (10-5-72). Astorga (14-5-72). En campos de cultivo.

Subcosmopolita.

Cheiranthus cheiri L.

León (5-6-73), en los muros de la Cárcel Vieja. San Pedro de Montes (POURRET). Euroasiática.

Matthiola fruticulosa (L.) Maire

Matthiola tristis (L.) R. Br.

Puente de Villarente (23-5-70). En pedregales cerca del río. Taxon citado en el suroeste de la provincia y en El Bierzo.

Circunmediterránea.

Rorippa pyrenaica (Lam.) Reichenb.

Nasturtium pyrenaicum (Lam.) R. Br.

Valcabado del Páramo (14-5-72), en charcas. Riaño y Llánaves (LOSA). Portilla de la Reina e Isoba (I.N.I.A.).

Sureuropea. Isoeto-Nanojuncetea.

Roripa silvestris (L.) Besser

Nasturtium silvestre (L.) R. Br.

Lago de Carucedo (12-5-73).

Europea.

Cardamine resedifolia $\mathrm{L}$.

Torrestío (10-6-73), en lugares encharcados.

Orófila alpina. 


\section{Arabis alpina $\mathrm{L}$.}

Vegarada (17-7-70). Castillo de Cornatel (14-4-72). Valporquero (19-8-72). Puebla de Lillo, Susarón (1-7-73). Hoces de Valdeteja (18-6-72). En rocas calizas. Muy citado en La Montaña y El Bierzo.

Arabis glabra (L.) Bernh.

A. perfoliata Lam. Turristis glabra L.

Torrestío (10-6-73), en hayedos. Varias citas en La Montaña y una en San Pedro de Montes (POURRET).

Circumboreal. De Populetalia albae.

Arabis hirsuta (L.) Scop.

Vegas del Condado (5-6-70). Nocedo (4-6-72). Crémenes (11-6-72). En desmontes cerca de la carretera. Riaño y Prioro (I.N.I.A.). San Pedro de Montes (POURRET). Circumboreal.

\section{Arabis nova Vill.}

A. saxatilis All. A. auriculata Lam.

Puerto de San Isidro (17-6-73), en calizas. Riaño (LOSA ESPAÑA); Oseja de Sajambre (I.N.I.A.).

Euromediterránea.

Arabis sagittata (Bertol.) DC.

Puebla de Lillo (1-7-73), en calizas. Nocedo (BORJA). Valdoré y Busdongo (I.N.I.A.).

Sur de Europa.

\section{Arabis stenocarpa Boiss./Reuter}

Valencia de D. Juan (4-5-73), en bosque mixto de Quercus ilex L. subsp. rotundifolia Lam. y Quercus faginea Lam.

San Esteban de Valdueza (LAfNZ).

Ibérica.

Arabis stricta Hudson

A. scabra All.

Vega de Gordón (31-5-73). Aviados (4-6-73). Santa Lucía (31-5-73). Siempre en calizas.

Orófila sureuropea.

Teesdalia coronopifolia (J. P. Bergeret) Thell.

T. lepidium DC.

Castrocalbón (25-3-73), en campos de cultivo.

Circunmediterránea.

Thlaspi arvense $\mathrm{L}$.

Virgen del Camino (12-5-73), en escombros cerca de la carretera. San Pedro de Montes (POURRET). Riaño (LOSA). Portilla de la Reina (I.N.I.A.).

Circumboreal. 


\section{Aethionema pyrenaicum Boutigny}

Vega de Gordón (31-5-73), en pedregales calcáreos. No encontramos en la bibliografía consultada ninguna cita de esta especie, probablemente por ser incluidas en A. saxatile (L.) R. Br.

Pirineos.

Alyssum granatense Boiss./Reuter.

De Geras a Pola de Gordón (29-6-71), en calizas.

Endemismo ibérico.

Neslia paniculata (L.) Desv.

Vogelia paniculata (L.) Hornem.

Villasabariego (11-6-72). Fuentes de Carbajal (18-5-74). En cultivos.

Paleotemplada. Secaletalia.

Ionopsidium prolongoi (Bss.) Batt., subsp. abulense (Pau) Laínz.

Ionopsidium abulense (Pau) Rothm.

Luyego (18-3-73), en campos de cultivo. Ferradillo, cerca de Ponferrada (LEROY/ LAINZ).

Ibérica.

Teesdaliopsis conferta (Lag.) Rothm.

Iberis conferta Lag.

Peña Cuiña, Ancares (25-7-70). Catoute (26-7-73), sobre sustrato silíceo. Citada también en La Montaña y El Bierzo.

Endemismo ibérico.

Iberis pruitii Tineo

I. tenoreana DC. I. lagascana DC.

Crémenes (11-6-72), en calizas.

Pirineos y Cordillera Cantábrica. Thlaspeetea rotundifolii.

Biscutella auriculata L.

Fuentes de Carbajal (18-5-74). Citas, no muy numerosas en La Montaña.

Mediterránea. Secalinetea.

Cardaria draba (L.) Desv.

Lepidium draba $\mathrm{L}$.

Valencia de D. Juan (23-5-74), cerca del Castillo.

Mediterránea. Secalinetalia.

Lepidium perfoliatum $\mathrm{L}$.

Herreros de Jamuz (6-5-73). Borde de camino. La Bañeza (LANGE).

Euroasiática.

Conringia orientalis (L.) Dumort

Ardoncino (27-4-74), en campos de cultivo arcillosos.

Sur de Europa. Secalinetea.

Brassica nigra (L.) Koch,

Valcabado del Páramo (14-5-72). Puente Castro (11-6-72). En sembrados. Fabero (I.N.I.A.).

Subcosmopolita. De Secalinetalia. 


\section{Sinapis arvensis $\mathrm{L}$.}

Villasabariego (11-6-72). Puente Castro (14-6-72), en cultivos. San Pedro de Montes (POURRET).

Cosmopolita.

Rhynchosinapis cheiranthos (Vill.) Dandy

subsp. cheiranthos

Brassica cheiranthos Vill.

Puerto de San Isidro (16-6-73). Peña Cuiña, Ancares (25-7-70). Citado en El Bierzo y zona montañosa nororiental.

Europa occidental.

Rhynchosinapis pseuderucastrum (Brot.) Franco

subsp. setigera (Gay ex Lange) Heywood

Brassica setigera Gay

Puente de Domingo Flórez (23-4-72). Villafranca del Bierzo (LANGE).

Ibérica.

Hirschfeldia incana (L.) Lagréze-Fossat

H. adpressa Moench

Barrio de Nuestra Señora (19-8-72). Puente Villarente (22-10-72). Astorga (13-5-73). Sur de Europa. Ruderal.

Rapistrum rugosum All.

subsp. rugosum

Villasabariego (11-6-72). Ardoncino (28-5-73). Siempre en sembrados.

Mediterránea. Puede llegar a ser cosmopolita.

\section{RESEDACEAE}

\section{Reseda phyteuma L.}

Villasabariego (22-10-72), en colinas arcillosas.

Circunmediterránea. Ruderal.

Sesamoides canescens (L.) O. Kuntze subsp. suffruticosus (Lange) Heywood Astrocarpus suffruticosus Lange

Castroquilame (13-5-73).

Ibérica.

Sesamoides pygmaea (Scheele) Kuntze subsp. minor (Lange) Heywood.

Astrocarpus sesamoides (L.) DC.

Peña Cuiña, Ancares (7-7-70). Citada también en La Montaña y Puerto de Manzanal. Mediterráneo occidental.

\section{CRASSULACEAE}

Sempervivum cantabricum J. A. Huber

Valporquero (22-7-73), sobre sustrato calcáreo.

Endemismo regional. 


\section{Sempervivum tectorum $\mathrm{L}$.}

Valporquero (19-8-72). Citado en Ambasmestas (LANGE).

Eurosiberiana.

Sedum hirsutum All.

subsp. hirsutum

Catoute (26-7-73), sobre sustrato silíceo. Se le ha citado en La Montaña y El Bierzo. Atlántica. Androsacetalia vandelii.

\section{SAXIFRAGACEAE}

Saxifraga canaliculata Boiss./Reuter

Valporquero (19-8-72). Camposolillo (29-6-73). Siempre sobre calizas. Muy citada en La Montaña.

Endemismo. Saxifragion trifurcato-canaliculatae.

\section{Saxifraga conifera Cosson/Durieu}

Valporquero (19-8-72). Puerto de San Isidro, Cebolledo (1-7-73). Santa Lucía (315-73). Siempre sobre sustrato calcáreo. Muy citada en La Montaña.

En Festucion burnatii.

Saxifraga dichotoma Sternb.

Subsp. albarracinensis (Pau) D. A. Webb

Val de San Lorenzo (18-3-73).

Endemismo ibérico.

Saxifraga spathularis Brot.

Alto de Leitariegos (26-6-70). Oseja de Sajambre (3-10-71). Puente de Domingo Flórez (15-5-72). Pinar de Puebla de Lillo (17-6-73). Pinar de Puebla de Lillo (RIVAS-MARTINEZ).

Europa occidental. Atlántica.

\section{Saxifraga tridactylites $\mathrm{L}$.}

Toral de los Vados (14-4-72).

Riaño (LOSA). Prioro (I.N.I.A.).

Mediterránea.

\section{Saxifraga trifurcata Schrader}

Puente de Domingo Flórez (16-5-72). Numerosas citas bibliográficas de La Montaña y El Bierzo.

Endemismo hispano:

\section{GROSSULARIACEAE}

\section{Ribes alpinum $\mathrm{L}$.}

La Uña (13-8-72). Cofiñal (13-8-72). Valporquero (19-8-72). Citas bibliográficas, poco numerosas, en La Montaña.

Orófila alpina. Quercetalia pubescentis. 


\section{ROSACEAE}

\section{Rubus saxatilis $\mathrm{L}$.}

Valporquero (19-8-72), en hayedos.

Eurosiberiana.

Rubus ulmifolius Schott.

Villafranca del Bierzo (14-4-72). Muy citado para la provincia.

Europa mediterránea.

Rosa pendulina L.

R. alpina $\mathrm{L}$.

Camposolillo (29-6-73). Puerto de San Glorio (6-8-72). Riaño (LOSA). Oseja de Sajambre (I.N.I.A.).

Orófila sureuropea.

Rosa villosa $\mathrm{L}$.

R. pomifera J. Herrmann

Cofiñal (13-8-72). Valporquero (19-8-72). León (VICIOSO).

Orófila euroasiática.

\section{Potentilla cinerea Chaix}

Picos de Europa, Valdeón (30-7-73).

Suroeste de Europa.

\section{Fragaria vesca $\mathrm{L}$.}

Puerto Ventana (17-6-71). Puebla de Lillo (1-6-71). Oseja de Sajambre (3-10-71). Villafranca del Bierzo (14-4-72). Siempre en sotobosque. Muy citado en La Montaña. Subcosmopolita. Fagetalia silvaticae.

Aphanes arvensis $\mathrm{L}$.

Astorga (14-5-72). San Pedro de Montes (POURRET). Sorribas (RIVAS MARTINEZ y Col.).

Mediterránea. Aphanion arvensis.

Sorbus aria (L.) Crantz

Cofiñal (13-8-72). Valporquero (19-8-72). Citado también en el centro y oriente de la montaña leonesa.

Europea. Querco-Fagetea.

Sorbus aucuparia $\mathrm{L}$.

Alto de Leitariegos (13-6-71). Cofiñal (13-8-72). Muy citado en La Montaña.

Europea. Querco-Fagetea.

Amelanchier ovalis Medicus

A. vulgaris Moench.

Valporquero (19-8-72). Castillo de Cornatel (23-6-72), en pedregales rocosos. Muy citado en La Montaña.

Europea mediterránea. Querco-Fagetea. 
Cotoneaster integerrimus Medicus

C. vulgaris Lindley

Valporquero (19-8-72), en calizas. Peña Corada (LOSA/MONTSERRAT). Macizo de Ubir̃ a (LAINZ).

Orófila alpina.

\section{LEGUMINOSAE}

\section{Genista florida L.}

Castroquilame (13-5-73). Citas bibliográficas, poco numerosas, de El Bierzo y Picos de Europa.

Endemismo ibérico.

Ulex minor Roth.

Puerto de las Señales (18-4-71). Puerto Ventana (19-6-71). Citas bibliográficas, no muy numerosas, en los puertos de León limítrofes con Asturias.

Atlántica. De Calluno-Ulicetea.

Lupinus hispanicus Boiss./Reuter

Ribaseca (3-6-73). En cultivos. Villafranca del Bierzo y Valcabado del Páramo (LANGE).

Ibérica.

Astragalus australis (L.) Lam.

Puerto de San Glorio (6-8-72).

Orófila alpina.

\section{Astragalus depressus $\mathrm{L}$.}

Nocedo (4-7-72). Crémenes (16-6-72). Lodares (29-6-72). Valporquero (19-8-72). Siempre sobre substrato calcáreo. Citas bibliográficas abundantes en el centro y este del tramo leonés de la Cordillera Cantábrica.

Mediterránea.

Astragalus sempervirens Lam.

subsp. catalaunicus (Br.-Bl.) Laínz.

San Emliiano (13-6-70), en desmontes cerca de la carretera. Torrebarrio (LAfNZ). Orófila sureuropea.

\section{Biserrula pelecinus $\mathrm{L}$.}

Pantano de Bárcena (12-5-73). León (COLMEIRO).

Circunmediterránea.

Vicia cracca $\mathrm{L}$.

Puente Orugo (30-6-71), en prados de siega. La Magdalena (2-7-71), en prados. Valporquero (19-8-72), en pastizal sobre sustrato calcáreo. San Pedro de Montes (POURRET). Riaño (LOSA). Portilla de la Reina y Oseja (I.N.I.A).

Euroasiática.

\section{Vicia disperma DC.}

La Magdalena (2-7-71). De Villafranca a Toral de los Vados (14-4-72). En herbazales al borde de caminos. Villafranca del Bierzo (LANGE).

Europa meridional y Argelia. 


\section{Vicia lutea $\mathrm{L}$.}

subsp. lutea

Vega de Espinareda (3-6-72), en colinas arcillosas.

Eurasia y Norte de Africa.

Vicia pyrenaica Pourr.

Collada del Viento, Vegarada (18-7-70), sobre sustrato calcáreo. Citas bibliográficas en la zona montañosa nororiental de la provincia.

Ibérica; Pirineos. Elyno-Seslerietea.

\section{Vicia sativa $\mathrm{L}$.}

subsp. nigra (L.) Ehrh.

V. pilosa Bieb. V. angustifolia L. V. cuneata Guss. V. heterophylla C. Presl.

Ribaseca (28-5-73). Vega de Espinareda (23-6-72). Citas bibliográficas en El Páramo y Bierzo, no muy numerosas.

Eurasia y Norte de África.

Lathyrus filiformis (Lam.) Gay

Valporquero (22-7-73).

Sur de Europa.

\section{Lathyrus setifolius $\mathrm{L}$.}

Vega de Gordón (31-5-73). San Pedro de los Montes (POURRET).

Mediterránea.

Melilotus indica (L.) All.

Melilotus parviflora Desf.

Puente de Villarente (22-10-72), en bordes de caminos.

Subcosmopolita. Artemisietea.

\section{Trigonella gladiata Steven}

Pola de Gordón (31-5-73), sobre calizas.

Euroasiática y mediterránea. Thero-Brachypodietea.

Trigonella polyceratia L.

Marne (21-6-69). Puente Castro (14-6-72). Vegas del Condado (7-5-73). En pastizales muy secos.

Mediterránea-Atlántica. Subruderal.

Medicago arabica All.

Parandones (14-4-72). Ponferrada (13-5-73). La Bañeza (LANGE).

Europa mediterránea.

Medicago ciliaris (L.) All.

San Juan de Paluezas (12-5-73).

Circunmediterránea.

Medicago orbicularis (L.) Bartal.

Villasabariego (11-6-72). Villafranca del Bierzo (LANGE).

Mediterránea. De Thero-Brachypodietea. 
Trifolium dubium Sibth.

Villafeliz de Babia (1-7-71). San Pedro de Montes (POURRET). Ponferrada (TUXEN/ OBERDORFER). Nocedo (BORJA). Vegamián (I.N.I.A.).

Europa.

Trifolium glomeratum L.

Herreros de Jamuz (6-5-73). Ribaseca (27-5-73). Cultivos de cereales en barbecho. Villafranca del Bierzo (LANGE).

Mediterránea-Atlántica. De Helianthemetalia.

Trifolium incarnatum $\mathrm{L}$.

Lago de Carucedo (20-5-73). León (VICIOSO).

Mediterránea.

Trifolium repens $\mathrm{L}$.

subsp. nevadense (Boiss.) D. E. Coombe

Puerto de San Glorio (6-8-72).

Sur de España.

Trifolium smyrnaeum Boiss.

T. lagopus Pourret

Garaño (16-6-73), en borde de camino. Villafranca del Bierzo (WILLKOMM/LANGE). Mediterráneo occidental.

Trifolium striatum $\mathrm{L}$.

Ribaseca (28-5-73), en borde de camino.

Euroasiática y mediterránea.

Trifolium strictum L.

T. laevigatum Poiret

Ponferrada (20-5-73). Prados húmedos. San Pedro de Montes (POURRET).

Mediterránea-Atlántica.

Trifolium subterráneum $\mathrm{L}$.

San Martín de Torres (12-5-72). San Martín del Camino (11-5-74). Sustrato silíceo.

San Pedro de Montes (POURRET). Villafranca del Bierzo (LANGE).

Mediterránea-Atlántica.

Trifolum tomentosum $\mathrm{L}$.

Ribaseca (28-5-73), en borde de caminos. San Pedro de Montes (POURRET).

Circunmediterránea.

\section{Lotus corniculatus $\mathrm{L}$.}

Vegas del Condado (25-6-68). Villargusán (26-6-69). Villafeliz de la Sobarriba (7-570). Vegamián (27-7-70). Llamas de Rueda (9-5-71). Lillo del Bierzo (6-6-71). Santibáñez de Bernesga (20-6-72).

Paleotemplada. 


\section{Lotus uliginosus Schkuhr}

Vegas del Condado (11-6-69). Villafeliz de la Sobarriba (11-7-70). Sena de Luna (30-6-71). Riaño (LOSA).

Euroasiática. Arrhenatherion.

\section{Anthyllis cornicina L.}

Herreros de Jamuz (28-5-73), en campos de cultivo. Valcabado del Páramo (LANGE). Ibérica.

\section{Anthyllis lotoides $\mathrm{L}$.}

Herreros de Jamuz (6-5-73), en campos de cultivo. Citada, como la anterior, en Valcabado del Páramo. (LANGE).

Ibérica.

\section{Anthyllis vulneraria $\mathrm{L}$.}

subsp. ibérica (W. Becker) Jallas

Villargusán (26-6-69). Vegas del Condado (24-6-67). Cistierna (12-6-69). Puente de Domingo Flórez (5-5-72). Siempre en pastizales.

var asturica: Valporquero (19-8-72). Vega de Gordón (31-5-73). Camposolillo (296-73).

Ibérica y Mediterránea.

Coronilla mínima L.

La Candamia (15-6-69). Riaño (12-6-69). Pola de Gordón (31-5-73).

Mediterránea. Ononido-Rosmarinetea.

Hippocrepis comosa $\mathrm{L}$.

San Juan de Paluezas (12-5-73). Santa Lucía (31-5-73). Sobre calizas. Entre Riaño y Cistierna (LOSA/MONTSERRAT). San Pedro de Montes (POURRET). Castro (LANGE). Pantano de Luna (RIVAS MARTINEZ, IZCO y COSTA).

Mediterránea. Festuco-Brometea.

\section{OXALIDACEAE}

\section{Oxalis corniculata L.}

León (20-5-73). Fabero (I.N.I.A.).

Cosmopolita.

\section{GERANIACEAE}

\section{Geranium rotundifolium L.}

Vegacervera (25-5-70). San Pedro de Montes (POURRET). Villafranca del Bierzo (LANGE).

Mediterránea-Atlántica. Hordeion.

Erodium cicutarium (L.) L'Hér.

Vegas del Condado (8-4-69). Vilecha (8-3-70). Robledo de Torío (8-3-70). De Villafranca a Toral de los Vados (14-4-72). Siempre en bordes de caminos. Numerosas citas bibliográficas en toda la provincia.

Subcosmopolita. Chenopodietalia. 


\section{Erodium malacoides (L.) L'Hér.}

Villafranca del Bierzo (14-4-72). Valencia de D. Juan (23-5-74). Ruderal. San Pedro de Montes (POURRET).

Euromediterránea.

Erodium petraeum (Gouan) Willd.

subsp. glandulosum (Cav.) Bonnier

Pantano del Porma (16-7-71). Puebla de Lillo (1-9-71). Sustrato calizo. Citas bibliográficas, no muy numerosas, en La Montaña y El Bierzo.

Pirineos y Norte de España. Thlaspeetea.

\section{EUPHORBIACEAE}

\section{Mercurialis perennis $\mathrm{L}$.}

Crémenes (16-6-72). Lodares (29-6-72). Valporquero (19-8-72). Citado en La Montaña y El Bierzo.

Europea. Querco-Fagetea.

\section{Euphorbia amygdaloides L.}

Villafranca del Bierzo (14-4-72). Vegas del Condado (15-5-73). Citas bibliográficas, no muy numerosas en La Montaña y El Bierzo.

Euroasiática. Querco-Fagetea.

\section{Euphorbia exigua L.}

Miñera de Luna (13-6-70). San Juan de Paluezas (12-5-73). Herreros de Jamuz (3-6-73). Citas bibliográficas, no muy numerosas, en La Montaña y El Bierzo.

Mediterránea. Thero-Brachypodion.

Euphorbia nicaensis All.

subsp. nicaensis

Puente de Domingo Flórez (23-4-72). Pantano de Bárcena (12-5-73).

Mediterráneo occidental. Rosmarinetalia.

\section{Euphorbla peplus L.}

Valencia de D. Juan (12-4-74), en cultivos.

Subcosmopolita.

\section{Euphorbia pinea L.}

E. segetalis L. subsp. pinea (L.) Hayek

Puente de Domingo Flórez (23-4-72). Carucedo y Villadepalos (LANGE).

Europa mediterránea.

\section{Euphorbia polygalifolia Boiss./Reuter}

Crémenes (16-6-72), en pedregales calizos. Citada para la zona nororiental de la provincia.

Ibérica. 


\section{RUTACEAE}

\section{Ruta montana (L.) L.}

San Juan de Paluezas (12-5-73), sobre sustrato calcáreo. Carucedo y Villafranca del Bierzo (LANGE).

Circunmediterránea.

\section{POLYGALACEAE}

\section{Polygala edmundii Chodat}

Catoute (26-7-73), sobre sustrato silíceo. Peña de Busdongo y Macizo de Ubiña (LAfNZ).

Endemismo hispano.

Polygala serpyllifolia Hose

Bemllera (20-6-70), en brezales. Valverde de Curueño (18-6-72). Muy citada en La Montaña.

Atlántica. De Seslerietalia coeruleae.

\section{ANACARDIACEAE}

\section{Pistacia terebinthus $\mathrm{L}$.}

Castillo de Cornatel (14-4-72). San Juan de Paluezas (12-5-73). Citas bibliográficas en El Bierzo y zona montañosa del nordeste provincial.

Circunmediterránea. Quercion ilicis.

\section{ACERACEAE}

\section{Acer pseudoplatanus $\mathrm{L}$.}

Fombasallá (20-7-73). Villafranca del Bierzo (LANGE). Retuerto (GUINEA). Posada de Valdeón (Instituto Nacional de Investigaciones Agrarias, I.N.I.A.).

Europea.

\section{CELASTRACEAE}

Evonymus vulgaris Scop.

E. europaeus L. pro part.

Devesa de Curueño (15-11-73), en un encinar. Garganta del río Cares (LASCOMBES). Riaño (LOSA).

Eurosiberiana. Populion albae.

\section{RHAMNACEAE}

\section{Rhamnus cathartica L.}

Puebla de Lillo, Susarón (1-7-73), sobre sustrato calcáreo. Citas bibliográficas, numerosas en la provincia.

Eurosiberiana. 
Frangula alnus Miller

Rhamnus frangula L.

Burbia (26-7-70). Citas bibliográficas, numerosas en La Montaña.

Eurosiberiana.

Rhamnus pumilus Turra

subsp. legionensis Rothm.

Puente de Domingo Flórez (25-6-72), sobre rocas dolomíticas. Bierzo (ROTHMALER). Nocedo (BORJA). Vegamián (I.N.I.A.).

Endemismo regional. Saxifragion trifurcato-canaliculatae.

\section{MALVACEAE}

Malva tournefortiana L.

Puente de Villarente (22-10-72), en borde de carretera.

Suroeste de Europa.

\section{THYMELAEACEAE}

Thymelaea tinctoria (Pourret) Endl.

Passerina tinctoria Pourret

El Castrillino (5-5-74), en bosquete de enebros.

España y Francia.

\section{GUTTIFERAE}

Hypericum hirsutum $L$.

Puerto Ventana (2-7-68). Catoute (26-7-73). Citas bibliográficas en La Montaña y Bierzo.

Subcosmopolita.

Hypericum pulchrum L.

Puerto Ventana (2-7-69). Catoute (26-7-73). San Pedro de Montes (POURRET). Cubillos (LANGE). Garganta del río Cares (LASCOMBES).

Mediterránea-Atlántica. Quercion roboris.

Hypericum tetrapterum Fries

Burbia (26-7-70). Lodares (29-6-72). Cofiñal (29-6-72). Lugares húmedos. Busdongo (LAGASCA). Riaño (LOSA).

Mediterránea-Atlántica. Molinio-Holoschoenion.

\section{VIOLACEAE}

Viola alba Besser

Castillo de Cornatel (14-4-72), sobre dolomías.

Europa central y meridional.

\section{Viola odorata L.}

Vegas del Condado (29-3-72), en un encinar. Citas bibliográficas muy numerosas en la provincia.

Eurasia y Mediterráneo. Populetalia albae. 


\section{CISTACEAE}

Cistus psilosepalus Siweet

Cistus hirsutus Lam.

Valsemana (22-7-70). Rodanillo (17-6-71). Pantano de Bárcena (13-5-73). Citas bibliográficas en El Bierzo y Maragatería.

Ibérica. Cistion hirsuti.

Cistus obtusifolius Sweet.

Cistus hirsutus Lam. X C. salvifolius L.

Lillo del Bierzo (6-6-71), mezclado con brezos.

Cistion hirsuti.

Cistus populifolius $\mathrm{L}$.

Pantano de Bárcena (12-5-73).

Región mediterránea occidental.

Halimium viscosum (Willk.) P. Silva

Puente Castro (28-6-70). Virgen del Camino (12-5-73).

Ibérica.

Helianthemum cinereum (Cav.) Pers.

H. rubellum Presl. $H$. paniculatum Dunol

Puente Castro (14-6-70), en colinas arcillosas muy soleadas.

Mediterráneo occidental.

Helianthemun croceum (Desf.) Pers.

Vegarada (19-7-70). Muy citado en La Montaña.

Mediterránea occidental.

Helianthemum hirtum (L.) Miller

Herreros de Jamuz (2-6-73). Puente Castro (6-6-73). Puente Castro (GANDOGER). Ibero-Provenzal.

Helianthemum pilosum (L.) Pers.

Herreros de Jamuz (27-4-74), en colinas secas arcillosas. Citas bibliográficas en el suroeste provincial, no muy numerosas.

Región mediterránea occidental. Thero-Brachypodietea.

Fumana procumbens (Dunal) Gren/Godron

Lodares (29-6-72), sobre sustrato calcáreo. Citas bibliográficas relativamente abundantes en La Montaña.

Submediterránea.

\section{LYTHRACEAE}

\section{Lythrum hyssopifolia L.}

Puente de Domingo Flórez (23-6-72). Zonas encharcadas. Cubillos, Bierzo (LANGE). Subcosmopolita. 


\section{ONAGRACEAE}

Epilobium anagallidifolium Lam.

E. alpinum auct. non $\mathrm{L}$.

Puerto de San Glorio (6-8-72). Macizo de Ubiña (LAINZ).

Boreal ártico-alpina.

Epilobium obscurum Schreber

Puerto de San Glorio (8-8-72). Catoute (26-7-73). Zonas húmedas de altura. Relativamente abundante en la zona montañosa nororiental.

Epilobium parviflorum Schreber

La Uña (13-8-72), en borde de acequia. San Pedro de Montes (POURRET). Villafranca del Bierzo (LANGE).

Paleotemplada.

Epilobium tetragonum L.

subsp. tournefortii (Michalet) Leveillé.

Puerto de San Glorio (6-8-72), en zonas muy húmedas. San Pedro de Montes (POURRET). Nogarejas, Tabuyo del Monte, Torneros de la Valdería (I.N.I.A.).

Mediterránea occidental.

\section{HALORAGACEAE}

\section{Myriophyllum spicatum L.}

San Justo de la Vega (14-5-72). Lago de Carucedo (20-5-74). Siempre sumergida en agua.

Circumboreal.

\section{CORNACEAE}

Cornus sanguinea L.

Ponferrada (13-5-73). Lorenzana (31-5-73). Siempre en bordes de fincas. Citada en El Bierzo y La Montaña.

Europa. Querco-Fagetea.

\section{UMBELLIFERAE}

Anthriscus silvestris (L.) Hoffn.

Catoute (26-7-73). Vegas del Condado (1-7-73). Citada en El Bierzo.

Anthriccus vulgaris Pers.

Gradefes (12-6-69). Santa Lucia (31-5-73).

Eurosiberiana.

Scandix australis $\mathrm{L}$.

subsp. microcarpa (Lange) Thell.

San Juan de Paluezas (12-5-73), sobre sustrato calcáreo. Villafranca del Bierzo (LANGE).

Circunmediterránea. 


\section{Smyrnium olusatrum L.}

Villafranca del Bierzo (14-4-72). San Juan de Paluezas (12-5-73). Villafranca del Bierzo (LANGE).

Mediterránea-Atlántica.

Pimpinella major (L.) Hudson

P. magna L.

Peña Cuiña, Ancares (25-7-70). Valporquero (19-8-72). Citas bibliográficas en El Bierzo y en el nordeste provincial.

Europea.

Delhavia tenuifolia Endl.

Nocedo (4-6-72), en pastizales calizos de altura. Citas bibliográficas en el nordeste montañoso provincial.

Ibérica. Elyno-Seslerietea.

Seseli libanotis (L.) Kosch.

subsp. libanotis

Valporquero (19-8-72). La Uña (29-8-72), sobre sustrato calcáreo. Citas bibliográficas en la mitad oriental de la montaña leonesa.

Euroasiática.

\section{Seseli nanum Dufour}

Valporquero (19-8-72), sobre sustrato calcáreo. Rabanal y Láncara de Luna (LAfNZ). Sabinares de Barrios de Luna (RIVAS-MARTínEZ, IZCO y COSTA).

Pirineos, Cordillera Cantábrica, Argelia.

\section{Oenanthe crocata L.}

var. tenuisecta Merino

Salentinos (26-7-73), en borde de arroyo. Citas bibliográficas en La Montaña y El Bierzo.

Atlántica. Phragmitetea.

\section{Foeniculum vulgare Gärtn.}

subsp. piperitum (Ucria) Coutinho.

Villafranca del Bierzo (14-4-72). Muy citado en la provincia.

Subcosmopolita.

Physospermum cornubiense (L.) DC.

$P h$. aquilegifolium Koch.

Catoute (26-7-73). Las Médulas (LANGE). Nocedo y Valdorria (CAMARA NIÑO). Nocedo (BORJA). Puebla de Lillo (RIVAS-MARTINEZ).

Atlántica. Quercetea robori-petreae.

\section{Conium maculatum L.}

Toral de los Vados (14-4-72). Mansilla de las Mulas (11-6-72). Astorga (12-5-73). Muy citado en la provincia.

Cosmopolita. Cheopodietalia. 


\section{Hohenackeria polyodon Cosson/Durieu}

San Juan de Paluezas (12-5-73). Sobre sustrato calcáreo.

España y Norte de África.

\section{Bupleurum rotundifolium $\mathrm{L}$.}

Ardoncino (28-5-73), en cultivos.

Subcosmopolita.

Trinia glauca (L.) Dumort

subsp. glauca

T. vulgaris DC.

Lugán (5-6-69). Aviados (5-6-72). En colinas calcáreas.

Orófila mediterránea. Ononido-Rosmarinetea.

Carum verticillatum Koch.

Sena de Luna (30-6-71). Cofiñal (13-8-72). Siempre en prados húmedos. Citas bibliográficas en El Bierzo y zona montañosa nororiental.

Atlántica. Molinio-Juncetea.

\section{Pastinaca sativa L.}

Palazuelo de Boñar (29-6-73), en herbazales cerca de la carretera. Riaño (LOSA). Portilla de la Reina y Riaño (I.N.I.A.).

Eurosiberiana. Populetalia albae.

\section{Heracleum sphondylium L.}

Fermín (29-6-69). Puebla de Lillo (5-7-70). Villafeliz de Babia (1-7-71). Valporquero (19-8-72). Citas bibliográficas en La Montaña y El Bierzo.

Mediterránea-Submediterránea.

\section{Laserpitium latifolium $\mathrm{L}$.}

Cofiñal (13-8-72). La Uña (13-8-72). Valporquero (19-8-72). Cerca de hayedos. San Pedro de los Montes (POURRET). Valdeteja (CÁMARA NIÑO).

Europea.

\section{Laserpitium nestleri Soyer-Villemet}

Valporquero (19-8-72). La Uña (13-8-72). Vega de Gordón (31-5-73). Sustrato calcáreo. Citas bibliográficas en el nordeste provincial no muy numerosas.

Pirineos e Ibérica y Cantábrica.

\section{Laserpitium siler $\mathrm{L}$.}

subsp. siler

La Uña (13-8-72), sobre calizas. Citas bibliográficas en La Montaña leonesa, no muy abundantes.

Orófila sureuropea.

\section{Caucalis platycarpos $\mathrm{L}$.}

Caucalis daucoides $\mathrm{L}$.

Puente Villarente (19-10-72), en cultivos. Riaño (LOSA). Villadangos (LAGASCA). Portilla de la Reina, Burón y Riaño (I.N.I.A.).

Submediterránea y Subatlántica. De Secalinetea. 
Turgenia latifolia (L.) Hoffm.

Caucalis latifolia $\mathrm{L}$.

Ardoncino (28-5-73), en cultivos.

Euroasiática.

\section{PRIMULACEAE}

Primula vulgaris Hudson

subsp. vulgaris

P. acaulis (L.) Hill.

Castillo de Cornatel (15-4-72). Puente de Domingo Flórez, Peña Rubia (23-4-72). Sobre sustrato dolomítico. Citas bibliográficas en La Montaña y El Bierzo.

Mediterránea. Atlántica.

Lysimachia ephemerum L.

Vegas del Condado (18-7-72).

Endemismo ibérico. Holoschoenetalia.

Asierolinon linum-stellatum (L.) Duby

Asterolinon stellatum Hoff./Link.

Sorribos de Alba (29-6-70). Astorga (12-5-73). En zonas arcilloso-silíceas. San Pedro de Montes (POURRET). Villafranca del Bierzo (LANGE). Nocedo (BORJA). La Robla y Rodiezmo (I.N.I.A.).

Mediterránea. Thero-Brachypodion.

\section{PLUMBAGINACEAE}

Armeria ciliata Lange

subsp. dasyphylla Lange

Luyego (1-5-73). Pantano de Bárcena (12-5-73). Existe en la provincia una cita imprecisa de LANGE.

Ibérica.

Ameria duriaei Boiss.

A. ancarensis Merino

Peña Cuiña, Ancares (25-7-70). Castrocontrigo (6-5-73). Castroquilame (13-5-73). Nocedo (BORJA). Arvas (RIVAS GODAY/RIVAS MARTINEZ). Vegamián (I.N.I.A.). Ibérica.

Armeria langei Boiss.

subsp. dareani (Continho) P. Silva.

Valporquero (19-8-72). Puerto de San Glorio (6-8-72). Citas bibliográficas poco abundantes. Alguna esporádica en El Bierzo y zona montañosa del nordeste de la provincia.

Ibérica.

Armeria maritima (Miller) Willd.

subsp. alpina (Willd.) P. Silva

A. cantabrica Boiss./Reuter

Camposolillo (29-6-73). Vegarada (18-7-70). Catoute (26-7-73). Siempre en calizas. Citas bibliográficas abundantes en el nordeste provincial.

Sureuropea. 
Armeria maritima (Miller) Willd.

subsp. eumaritima

Puente de Domingo Flórez (6-5-72).

var. Caballeroi: Astorga (15-5-72).

var. Merinoi: Peña Cuiña, Ancares (25-7-70).

Citas bibliográficas en El Bierzo y Maragatería, no muy abundantes.

Armeria segoviensis Gaud.

subsp. lacaitae (H. Villar) Borja

Ribaseca (3-6-73).

Ibérica.

Armeria transmontana (Samp.) Lawrence

Catoute (26-7-73).

Ibérica.

\section{OLEACEAE}

\section{Ligustrum vulgare L.}

Villafranca del Bierzo (14-4-72). Lorenzana (10-6-73). En setos bordeando fincas. Villafranca del Bierzo (LANGE). Ponferrada (COLMEIRO).

Euroasiática.

Phyllyrea angustifolia L.

Puente de Domingo Flórez (12-5-73), mezclada con matorral de encina. Pombriego (POURRET).

Mediterráneo occidental. Quercetea ilicis.

\section{GENTIANACEAE}

Centaurium erythraea Rofn.

subsp. grandiflorum (Biv.) Melderis

Peña Rubia, Puente de Domingo Flórez (23-6-72).

Europea y circunmediterránea.

Gentiana angustifolia Vill.

Picos de Europa (30-7-73).

Orófila alpina.

\section{APOCYNACEAE}

Vinca difformis Pourret, subsp. difformis

V. media Hoffmanns./Link

Puente de Domingo Flórez (23-4-72).

Mediterráneo occidental. 


\section{ASCLEPIADACEAE}

Vincetoxicum hirundinaria Medicus

V. officinale (L.) Moench

Vegacervera (11-7-70), sobre sustrato calcáreo. Citas bibliográficas en el nordeste de la provincia y en El Bierzo.

Eurasia y África septentrional.

\section{RUBIACEAE}

\section{Cruciata laevipes Opiz}

Galium cruciata Scop.

La Vecilla (5-6-71). Villafranca del Bierzo (14-4-72). Crémenes (10-5-72). Lago de Carucedo (12-5-73). Armada (8-6-74). San Pedro de Montes (POURRET). Villafranca del Bierzo (LANGE). Riaño (LOSA).

Europea.

\section{Galium murale All.}

Puente de Domingo Flórez (6-5-72). En dolomías.

Circunmediterránea.

Galium lucidum All.

Galium rigidum Vill.

La Uña (13-8-72), en substrato calcáreo. Entre Riaño y Cistierna (LOSA/MONTSERRAT). San Pedro de Montes (POURRET). Sierra de Manzanal (LANGE).

Europea.

\section{Galium rotundifolium $\mathrm{L}$.}

Burbia (27-7-70). Citas bibliográficas en La Montaña y El Bierzo, no muy numerosas. Orófila alpina. Fagion silvaticae.

Galium umbellatum Murray

Galium umbellatum Lam.

G. silvestre Poll.

Pantano del Porma (16-7-71). Puerto de San Glorio (6-8-72). Valporquero (19-8-72). Mirantes de Luna (10-6-73). Citas bibliográficas en el nordeste montañoso provincial.

\section{Rubia peregrina $\mathrm{L}$.}

Villafranca del Bierzo (14-4-72). Puente de Domingo Flórez (23-4-72). Siempre en encinares. San Pedro de Montes (POURRET). Cubillos (LANGE).

Submediterránea. Quercetea ilicis.

\section{CONVOLVULACEAE}

\section{Convolvulus lineatus $\mathrm{L}$.}

Villasabariego (14-6-72), en colinas arcillosas de exposición sur.

Circumboreal. Thero-Brachypodietea. 


\section{BORAGINACEAE}

Lithospermum officinale L.

Ponferrada (19-5-73).

Euroasiática. Populetalia albae.

Neatostema apulum (L.) I. M. Johnston

Lithospermum apulum (L.) Vahl

Destriana (28-5-73). Fuentes de Carbajal (25-5-74). En cultivos arcillosos.

Mediterránea. Thero-Brachypodion.

\section{Symphytum officinale $\mathrm{L}$.}

Salentinos (26-7-73), en campos de cultivo. San Pedro de Montes (POURRET).

Eurosiberiana.

Anchusa arvensis (L.) Bieb.

subsp. arvensis

Lorenzana (31-5-73). Borde de cultivo. Citada en el noroeste de la provincia y en El Bierzo.

Euroasiática. Secalinetea.

Myosotis alpestris F. W. Schmidt.

M. silvatica Hoffm.

Torrestío (10-6-73). Cebolledo, Puebla de Lillo (1-7-73). Hoces de Valdeteja (18-672). Citas bibliográficas en la zona montañosa del nordeste de la provincia.

Orófila paleotemplada.

Myosotis laxa Lehm.

subsp. caespitosa (Schultz) Hyl.

Myosotis caespitosa Schultz

Zotes del Páramo (14-5-72). En charcas. Entre Caldas de Nocedo y Oville (CAMA-

RA NIÑO).

Circumboreal. Isoeto-Nanojuncetea.

Myosotis ramosissima Rochel

subsp. ramosissima

M. hispida Schlecht.

Vegas del Condado (28-6-70). Herreros de Jamuz (6-5-73). Riaño (LAfNZ).

Europa, Asia Menor y Africa del Norte.

\section{CALLITRICHACEAE}

\section{Callitriche stagnalis Scop}

Villadepalos (14-4-72), en acequias.

Submediterránea-Subatlántica. Potamion eurosibiricum.

\section{LABIATAE}

\section{Ajuga pyramidalis $\mathrm{L}$.}

subsp. pyramidalis

Puebla de Lillo, Cebolledo (17-6-73). San Pedro de Montes (POURRET); Peña Prieta (LOSA).

Orófila sureuropea. Nardion. 
Ajuga reptans $\mathrm{L}$.

Crémenes (10-5-72), en prados. Citas bibliográficas en la zona montañosa nororiental de la provincia.

Euroasiática. En Querco-Fagetea.

Teucrium aragonense Losc./Pardo

subsp. expansum Pau

Vegacervera (11-7-70), sobre sustrato calcáreo.

Endemismo hispano. Ononido-Rosmarinetea.

Melitis melissophyllum L.

subsp. melissophyllum

Castillo de Cornatel (27-6-72). Pola de Gordón (1-6-74). Siempre nemoral. Citas bibliográficas numerosas en La Montaña.

Europea. Querco-Fegetea.

Phlomis herba-venti L.

subsp. herba-venti

Villasabariego (11-6-72), en colinas arcillosas. León (COLMEIRO).

Mediterráneo y Asia.

Galeopsis ladanum L.

Catoute (26-7-73). Ruderal. Citas bibliográficas en La Montaña y El Bierzo.

Euroasiática.

Stachys alpina L.

Valporquero (19-8-72), en hayedos. Riaño (LOSA). Nocedo (BORJA). Valdeteja (CÁMARA NIÑO).

Orófila alpina. Querco-Fagetea.

Stachys officinalis (L.) Trevisan

Betonica officinalis $\mathrm{L}$.

Vegamián (27-7-70). Cofiñal, Valle de Pinzón (21-7-74). Citas bibliográficas en La Montaña y El Bierzo.

Europea. Querco-Fagetea.

Nepeta latifolia DC.

Puebla de Lillo (29-7-73), en borde de camino. Riaño (LOSA).

Ibero-pirenaica.

Glechoma hederacea L.

Villadangos (14-4-72). Ponferrada (13-5-73). Siempre nemoral. Citas bibliográficas en La Montaña y El Bierzo.

Circumboreal. Querco-Fagetea.

Acinos alpinus (L.) Moench

Calamintha alpina (L.) Lam.

Santa Lucía (18-5-69). Sorribos de Alba (6-7-70). Lillo del Bierzo (6-6-71). Citas bibliográficas numerosas en La Montaña y El Bierzo.

Orófila alpina. En Elyno-Seslerietea. 
Calamintha sylvatica Bromf.

subsp. ascendens (Jordan) P. W. Ball.

Calamintha officinalis Moench

Sorribos de Alba (6-7-70). León (COLMEIRO).

Submediterránea-Subatlántica.

Clinopodium vulgare $\mathrm{L}$.

Calamintha clinopodium (L.) Moris

Venta de la Tuerta (22-5-70), en brezal. Puebla de Lillo, Susarón (1-7-73), en calizas. Riaño (LOSA).

Circumboreal. De Querco-Fagetea.

Origanum virens Hoffmanns./Link

Vegacervera (10-7-70). Lodares (29-6-72). Puente de Domingo Flórez (12-5-73). Sobre sustrato calcáreo. Carucedo, Cubillos y Villafranca del Bierzo (LANGE).

Ibérica. Puede considerarse como de Thlaspeetea.

Origanum vulgare $\mathrm{L}$.

Vega de Gordón (31-5-73), sobre calizas. Citas bibliográficas abundantes en la mitad norte de la provincia.

Euroasiática.

Thymus praecox Opiz

subsp. praecox.

Puebla de Lillo, Susarón (17-7-71), sobre sustrato calcáreo. No encontramos citas de esta subespecie, previa consulta de toda la bibliografía que, amablemente, puso a nuestra disposición el Prof. Jalas de la Universidad de Helsinki.

Centro y Oeste de Europa.

\section{Thymus vulgaris $\mathrm{L}$.}

Carbajal de la Legua (10-6-69). Valsemana (22-7-72). Numerosas citas bibliográficas en toda la provincia.

Mediterránea. Muy amplia en su comportamiento fitosociológico.

Mentha arvensis $\mathrm{L}$.

Miñera de Luna (1-7-71). En prados de siega. Riaño (LOSA). Prioro (I.N.I.A.).

Circumboreal. Isoeto-Nanojuncetea.

Mentha cervina Fresen

Presila cervina L.

Valcabado del Páramo (14-5-72), en charcas.

Mediterráneo occidental. Isoeto -Nanojuncetea.

\section{Horminum pyrenaicum $\mathrm{L}$.}

Cebolledo, Puerto de San Isidro (17-6-73). Picos de Europa (LASCOMBES).

Orófila alpina. 


\section{Datura stramonium L.}

Valcabado del Páramo (14-5-72). Villamañán (23-5-74). Siempre ruderal. Citas bibliográficas relativamente abundantes en El Páramo y Maragatería.

Subcosmopolita.

\section{SCROPHULARIACEAE}

Verbascum lychnitis $L$.

Valporquero (19-8-72), sobre sustrato calcáreo. Riaño y Babia (LAfNZ).

Eurosiberiana.

\section{Scrophularia alpestris Gay}

Valverde de Curueño (18-6-72). Citas bibliográficas numerosas en La Montaña.

Pirineos y Cordillera Cantábrica. Querco-Fagetea.

Scrophularia scorodonia L.

Villafranca del Bierzo (14-4-72).

Atlántica.

Antirrhinum braun-blanqueti Rothm.

Puebla de Lillo (1-7-73). Felmín (29-6-69). Citas bibliográficas abundantes en el nordeste provincial.

Ibérica.

Linaria faucicola Leresche/Levier

La Uña (13-8-72). Sustrato calizo. Citas bibliográficas en el nordeste de la provincia. Endemismo. Festucion burnatii.

Linaria saxatilis (L.) Chaz.

L. tournefortii (Poiret) Stendel

Santa María del Páramo (21-5-70). Benllera (7-6-70). Puente Villarente (28-10-72). Catoute (26-7-73). Siempre impurificando cultivos. Muy citada en la provincia.

Ibérica.

Verónica acinifolia $\mathrm{L}$.

Zotes del Páramo (14-5-72), en campos de cultivo de cereales.

Euroasiática.

\section{Verónica agrestis $\mathrm{L}$.}

Hoces de Valdeteja (18-6-72). Vegas del Condado (28-3-73). Vega de Gordón (315-73). Santa Lucía (31-5-73). Torrestío (10-6-73). San Pedro de Montes (POURRET). Villafranca del Bierzo (LANGE).

Circumboreal.

\section{Verónica anagallis-aquática $\mathrm{L}$.}

Lago de Carucedo (13-5-73). Vegas del Condado (1-7-74). Siempre en bordes de cauces de agua. San Pedro de Montes (POURRET). Villafranca del Bierzo (LANGE). Circumboreal. 
Verónica anagalloides Guss.

Lago de Carucedo (13-5-73). Riaño (LOSA).

Euroasiática y mediterránea. Isoeto-Nanojuncetea.

Verónica brevistila Moris

Val de San Lorenzo (18-3-73). Se aparta de V. verna L. (a la cual la subordina Flora Europaea) tanto por tener la cápsula muy pelosa, como por el hecho de poseer hojas menos divididas. Los sépalos, por otra parte, no sobrepasan al fruto.

Mediterráneo occidental.

Verónica tenuifolia Asso

Verónica conmutata Willk.

V. assoana (Boiss.) Willk.

Villafeliz de la Sobarriba (7-5-70). La Candamia (20-5-70). El Castrillino (20-5-72). Nocedo (BORJA).

España.

Verónica fruticulosa $\mathrm{L}$.

Vegarada (17-7-70). Coriscao (LOSA).

Orófila alpina. Caricetea curvulae.

Verónica polita Fries

Vegas del Condado (2-3-73), en la base de una pared de barro.

Paleotemplada. Chenopodietea.

Verónica serpyllifolia L.

subsp. humifusa (Dickson) Syme

V. humifusa Dickson; V. apenina Tausch

Aviados (4-6-72). Valverde de Curueño (18-6-72). Lodares (29-6-72). Colegiata de Arbas (11-6-73). Cebolledo, Puebla de Lillo (8-7-73).

Europea.

Verónica austriaca L.

subsp. teucrium (L.) D. A. Webb

Verónica teucrium L.

Utrero, Pantano del Porma (9-7-72), sobre sustrato calcáreo. Riaño (LOSA). Nocedo (BORJA). Peña del Valdorria (CÁMARA NIÑO). Vegamián (I.N.I.A.).

Eurosiberiana.

Verónica triphyllos $\mathrm{L}$.

Llamas de Rueda (9-5-71). Astorga (14-5-72). En campos de cultivo. Sorribos (RIVAS-MARTINEZ, IZCO y COSTA).

Eurasia y Norte de Africa.

Verónica verna $\mathrm{L}$.

Vilecha (8-3-70). Llamas de Rueda (9-5-71). En campos de cultivo. Nocedo (BORJA). Riaño, Macizo de Ubiña y Somiedo (LAINZZ). Puerto de Manzanal (TUXEN/ OBERDORFER).

Euroasiática. 
Tozzia alpina L.

Pinar de Puebla de Lillo (17-6-73). Sustrato silíceo y mucha humedad edáfica.

Orófila sureuropea.

Odontites longiflora (Vahl.) Webb

Valporquero (19-8-72), sobre sustrato calcáreo. Citas bibliográficas en La Montaña, no muy numerosas.

Ibérica. Ononido-Rosmarinetea.

Odontites verna (Bellardi) Dumort.

subsp. verna

O. rubra Besser

Valdevimbre (14-7-70). Truchas (8-8-71). Siempre en bordes de cultivos. Citas bibliográficas numerosas en La Montaña y El Bierzo.

Europea.

Pedicularis kerneri Dalla Torre

$P$. rostrata L. pro parte. $P$. rhaetica A. Kerner

Vegarada (18-7-70).

Alpes y Pirineos.

Pedicularis mixta Grenier/Godron

Puerto de San Glorio (6-8-72). Cofiñal (13-8-72). Catoute (26-7-73). Sustrato silíceo. Circo glaciar de Cubil del Can (LOSA).

Endemismo ibérico. Caricetea curvulae.

Pedicularis pyrenaica Gay

Fombasallá (20-7-73). Citas bibliográficas en La Montaña; no muy numerosas.

Endemismo pirenaico. Caricetea curvulae.

Rhinanthus angustifolius C. C. Gmelin

subsp. angustifolius

Rhinanthus major Ehrh.

Peña Cuiña, Ancares (25-7-70). Sena de Luna (30-6-71). Camposolillo (29-6-73).

Citas bibliográficas en La Montaña y El Bierzo, no muy numerosas.

Euroasiática.

\section{GLOBULARIACEAE}

Globularia cordifolia L.

Piedrafita, Cármenes (11-7-70). Citas bibliográficas numerosas en la zona montañosa nororiental.

Orófila. Centro y Sur de Europa.

\section{OROBANCHACEAE}

Orobanche caryophyllacea $\mathrm{Sm}$.

o. vulgaris Poiret

Torrestío (10-6-73). Picos de Europa, más bien en Asturias (LAfNZ).

Eurosiberiana. 


\section{LENTIBULARIACEAE}

Pinguicula grandiflora Lam.

subsp. grandiflora

Pinar de Puebla de Lillo (17-6-73). Citas bibliográficas en La Montaña, no muy numerosas.

Orófila alpina. Caricetalia fuscae.

Pinguicula vulgaris $\mathrm{L}$.

Cofiñal (13-8-72). Pedrosa (QUER). Montañas de León (LAGASCA). Nocedo (BORJA).

Europea. Caricetalia fuscae.

\section{PLANTAGINACEAE}

Plantago lagopus $\mathrm{L}$.

Benllera (29-6-70).

Euromediterránea.

\section{CAPRIFOLIACEAE}

\section{Viburnum lantana L.}

Villafranca del Bierzo (15-4-72). Valporquero (19-8-72). Citas bibliográficas en La Montaña y Bierzo.

Eurasia y África septentrional.

Lonicera etrusca Sant.

Villafranca del Bierzo (14-4-72). Puente de Domingo Flórez (23-4-72). Pantano de Bárcena (12-5-73). Citas bibliográficas en La Montaña y El Bierzo, poco numerosas. Sureuropea. Querco-Fagetea.

Lonicera periclymenum $\mathrm{L}$.

Villafranca del Bierzo (14-4-72). San Juan de Paluezas (12-5-73). Citas bibliográficas en La Montaña y El Bierzo.

Submediterránea-Subatlántica.

Lonicera xylosteum L.

Armada (11-7-73). Sustrato calcáreo. Riaño (LOSA).

Eurosiberiana. Querco-Fagetea.

\section{VALERIANACEAE}

Valeriana officinalis $\mathrm{L}$.

Cofiñal (13-8-72), en borde de arroyo; sustrato silíceo. Citas bibliográficas en La Montaña y El Bierzo.

Europa y Asia.

Centranthus ruber (L.) DC.

León (6-6-73), en las murallas de San Isidoro. Citas bibliográficas numerosas en la provincia.

Mediterránea-Atlántica. 


\section{DIPSACACEAE}

Succisa pratensis Moench

Scabiosa succisa L. Succisa praemorsa (Gilib.) Ascherson

Puente Orugo (30-6-71), en prados de siega. Riaño (LOSA). Prioro (I.N.I.A.).

Eurosiberiana. Molinio-Juncetea.

Knautia arvensis (L.) Coulter

Palazuelo de Boñar (29-6-73). Riaño (LOSA).

Eurosiberiana. Ononido-Rosmarinetea.

Scabiosa columbaria L.

Valporquero (19-8-72). Prados de León (LAGASCA). Carucedo (LANGE). Riaño (LOSA). Oseja de Sajambre (I.N.I.A.).

Mediterránea. Con cierta frecuencia en Ononido-Rosmarinetea.

\section{CAMPANULACEAE}

Campanula arvatica Lag.

subsp. adsurgens (Leresche/Levier) Damboldt

Campanula adsurgens Ler./Lev.

Peña Rubia, Puente de Domingo Flórez (14-4-72). El Bierzo (ROTHMALER).

Ibérica. En Saxifragion trifurcato-canaliculatae.

Campanula arvatica Lag.

subsp. arvatica

Valporquero (19-8-72). La Uña (13-8-72). Vega de Gordón (31-5-73). Camposolillo (29-6-73). Siempre en fisuras de rocas calizas. Numerosas citas bibliográficas en el nordeste de la provincia.

Endemismo ibérico. En Festucion burnatii.

Campanula cantábrica Feer.

La Uña (13-8-72), en calizas. Numerosas citas bibliográficas en la zona montañosa nororiental de la provincia.

Endemismo regional. En Xerobromion.

Campanula erinus $L$.

San Juan de Paluezas (12-5-73), en cultivos. Citas bibliográficas en El Bierzo y La Montaña; poco numerosas.

Euromediterránea.

Campanula lusitánica L.

Campanula loeflingii Brot.

Castroquilame (12-5-73), en borde de cultivos.

Ibérica.

Campanula recta Dulac

Campanula linifolia Lam.

Puerto de San Glorio (6-8-72), en sustrato silíceo.

Orófila alpina. Apeninos y Balkanes. 


\section{Campanula patuia L.}

subsp. patula

La Uña (13-8-72), cerca de hayedos. Citas bibliográficas en el nordeste de la provincia, poco numerosas.

Europea. Querco-Fagetea.

Campanula scheuchzeri Vill.

Puerto de San Glorio (6-8-72). San Pedro de Montes (POURRET). Picos de Europa (LASCOMBES). San Glorio y Tarna (LOSA). Isoba y Portilla de la Reina (I.N.I.A.). Orófila alpina.

Legousia castellana (Lange) Samp.

Specularia castellana Lange

Garaño (16-6-73). León (GANDOGER).

Ibérica.

Lobelia urens $\mathrm{L}$.

Vegas del Condado (19-7-72). Villarrasil (CÁMARA NIÑO).

Atlántica.

\section{COMPOSITAE}

Eupatorium cannabinum L.

subsp. cannabinum

Burbia (26-7-70). Lugares húmedos. San Pedro de Montes (POURRET). Villafranca del Bierzo (LANGE).

Euroasiática. Molinio-Holoschoenetalia.

Aster sedifolius L.

subsp. sedifolius

Valporquero (19-8-72), en calizas.

Sudeurosiberiana.

Erigeron acer L.

subsp. acer

Valporquero (19-8-72), sobre sustrato calcáreo. San Pedro de Montes (POURRET).

Viadangos (LAGASCA). Puente de las Palomas, Piedrafita de Babia (LAfNZ).

Circumboreal.

Logfia arvensis (L.) J. Holub

Filago arvensis L.

Profusamente citada en la provincia. San Justo de la Vega (14-5-72). Ribaseca (285-73).

Eurosiberiana. Secalinetea.

Bombycilaena discolor (Pers.) Laínz

Micropus bombycinus Lag.

Miñera de Luna (1-7-71). Puente Castro (11-6-72).

Circunmediterránea.

Omalotheca sylvatica (L.) Schultz

Gnaphalium silvaticum L.

Pefia Cuiña, Ancares (25-7-70). Catoute (26-7-73). Fombasallá (30-7-73). Muy citada en La Montaña.

Circumboreal. 


\section{Gnaphalium luteo-album L.}

Valcabado del Páramo (12-5-72). Pola de Gordón (COLMEIRO).

Subcosmopolita.

Helichrysum italicum (Roth) G. Don fil.

subsp. serotinum (Boiss.) P. Fourn.

Santa María del Condado (15-8-72), en ribazos arcillosos.

Mediterránea. Aphyllantion.

Phagnalon saxatile Cass.

Puente de Domingo Flórez (25-5-72). En rocas dolomíticas. Villafranca del Bierzo (LANGE).

Mediterráneo occidental.

Inula montana L.

Vegacervera (10-7-70). Pantano del Porma (16-7-71). Siempre en calizas. Citas bibliográficas, no muy numerosas, en La Montaña.

Mediterránea-Atlántica. Ononidetalia striatae.

Chamaemelum mixtum (L.) All.

Anthemis mixta L.

Puente de Villarente (20-10-72), en campos de cultivo. Citada en el Puerto de Manzanal (TUXEN/OBERDORFER).

Europa mediterránea. Thero-Bromion.

Chamaemelum nobile (L.) All.

Anthemis nobilis $\mathrm{L}$.

Sena de Luna (30-6-71), en prados de siega. Herreros de Jamuz (6-5-73).

var. discoidea Boiss. (Anthemis aurea DC.).

En Vegas del Condado (24-4-73), cerca del río, en pedregales. La especie se encuentra abundantemente citada en toda la provincia.

Atlántica. De Isoeto-Nanojuncetea.

Chrysanthemum pallidum Lag.

subsp. pallidum var. pallidum

Puente Villarente (20-5-70). En pedregales secos cerca del río Porma.

Ibérica.

Chrysanthemum segetum L.

Castroquilame (12-5-73), en cultivos. Villafranca del Bierzo (LANGE). San Pedro de Montes (POURRET).

Subcosmopolita.

\section{Tanacetum vulgare $\mathrm{L}$.}

Valporquero (19-8-72). Ponferrada (COLMEIRO). Puerto Ventana, Sahélices del Río y Sahagún (I.N.I.A.).

Eurosiberiana. 
Leucanthemopsis pallida (Miller) Heywood

subsp. pallida

Pyrethrum hispanicum Willk.

Puente Villarente (10-4-69). Vegas del Condado (31-3-70). Astorga (20-4-73). Citada además en la zona central de la provincia.

Endemismo ibérico. Helianthemetea.

Artemisia absinthium L.

Garrafe de Torio (18-6-72), en bordes de carretera. Citada ampliamente en La Montaña y comarcas limítrofes.

Mediterránea. Chenopodietalia.

Artemisia campestris L.

subsp. glutinosa (Gay) Batt.

Puente Castro (11-6-72). Cerros de Lancia (22-10-72). Puente de Domingo Flórez (5-5-72). Siempre en cultivos y bordes de caminos.

Circumboreal.

Arnica montana L.

subsp. atlantica A. Bolós.

Catoute (26-7-73). Zonas silíceas. Muy citada en toda la montaña leonesa.

Orófila centroeuropea.

Doronicum austriacum Jacq.

Catoute (26-7-73). Picos de Europa (LASCOMBES). Arbas (LAGASCA). Leitariegos (ALLORGE).

Orófila alpina.

Doronicum pardalianches $\mathrm{L}$.

Castillo de Cornatel (14-4-72). San Pedro de Montes (POURRET).

Mediterránea-Atlántica. Fagetalia silvaticae.

Senecio doria L.

Miñera de Luna (1-7-71).

Europea.

Senecio erucifolius L.

Valporquero (19-8-72).

Euroasiática.

Senecio lividus $L$.

Herreros de Jamuz (5-5-73). Villadangos (LAGASCA). Villafranca del Bierzo (LANGE).

Circunmediterránea.

Senecio minutus (Cav.) DC.

Pantano del Porma (16-7-71). Vega de Gordón (31-5-73). Rabanal de Luna y Las Bodas (LAfNZ).

Endemismo ibérico. Pastizales subumbrosos de Thero-Brachypodietalia. 


\section{Calendula arvensis $\mathrm{L}$.}

Villafranca del Bierzo (14-4-72), en cultivos.

Submediterránea.

\section{Carlina corymbosa $\mathrm{L}$.}

subsp. corymbosa

Puente de Villarente (22-10-72), en cultivos abandonados. La Chana (POURRET).

Circunmediterránea.

\section{Carlina vulgaris L.}

Cofiñal (13-8-72), en pastizales ácidos. Abundantes citas bibliográficas en La Montaña.

Eurosiberiana.

\section{Atractylis humilis $\mathrm{L}$.}

Sorribos de Alba (6-7-70), en colinas arcillosas. Montañas de León (COLMEIRO). Mediterránea occidental.

Carduus carlinifolius Lam.

Relativamente abundantes en la zona nororiental de la provincia. Collada del Viento, Vegarada (18-7-70).

Orófila alpina.

Carduus medius Gou.

Puerto de San Glorio (6-8-72). La Uña (13-8-72). Valporquero (19-8-72).

Ibérica.

Cirsium vulgare (Savi) Ten.

Cirsium lanceolatum Scop.

Castroquilame (13-5-73), en borde de camino. Citada en Riaño por VICIOSO.

Euroasiática. Artemisietea y Plantaginetea.

Cirsium rivulare (Jacq.) All.

Muy citado en el nordeste de la provincia.

Puente Orugo (30-6-71). Puerto de San Glorio (6-8-72). Siempre en prados húmedos. Del centro y sur de Europa. Montio-Cardaminetea.

Leuzea conifera DC.

Muy citado en La Montaña.

Pantano del Porma (22-6-74). Crémenes (16-6-72). Siempre sobre sustrato calcáreo. Mediterránea-Atlántica. Ononido-Rosmarinetea.

Centaurea vinyalsii Sennen

subsp. aproximata (Rouy) Dostál

Centaurea amara $\mathrm{L}$.

Armada (19-7-73), en bordes de carretera.

Eurosiberiana. Thero-Brachypodietea.

Centaurea collina L.

subsp. serratulifolia (Sennen/Pau) Hayek.

Camposolillo (29-6-73). Sustrato calcáreo.

Mediterránea. 


\section{Centaurea ornata Willd.}

Puente Villarente (22-10-72), en bordes de carretera. Citada en Puente Castro por GANDOGER.

\section{Centaurea scabiosa L.}

Abundantes citas bibliográficas en La Montaña.

Vegacervera (10-7-70). Puente Villarente (22-10-72).

Eurosiberiana. Secalinetalia.

Carduncellus monspeliensium All.

Valporquero (22-7-73), en calizas. Nocedo (BORJA); Vegaquemada, La Robla y Rodiezmo (I.N.I.A.).

Mediterránea. Thero-Brachypodietalia.

Tolpis barbata (L.) Gaertner

Villadangos (1-6-69). Virgen del Camino (11-6-69). Castro del Condado (11-7-70). Puente Villarente (29-10-72). En campos de cultivos ácidos.

Mediterránea. Helianthemetea.

Leontodon farinosum Merino/Pau

Puente de Domingo Flórez (13-5-72). En rocas dolomíticas. Bierzo (ROTHMALER). Endemismo regional. Saxifragion trifurcato-canaliculatae.

\section{Scorzonera graminifolia L.}

var. minor

Puente Castro (26-6-70), en cultivos abandonados. Villafranca del Bierzo (LANGE). España, Portugal y Marruecos. En etapas aclaradas de Quercion ilicis.

\section{Scorzonera hispanica L.}

Benllera (29-6-70), en brezales. León (COLMEIRO).

Eurosiberiana. Thero-Brachypoditalia.

\section{Scorzonera laciniala L.}

Podospermum laciniatum DC.

Virgen del Camino (12-5-73), en borde de carretera. Bierzo (POURRET).

Mediterránea. Thero-Brachypodietalia.

Sonchus asper Vill.

Puente de Villarente (22-10-72), en bordes de caminos. Villafranca del Bierzo (LANGE). Ponferrada (TUXEN/OBERDORFER).

Subcosmopolita. Chenopodietea.

\section{Sonchus oleraceus $\mathrm{L}$.}

De Villafranca del Bierzo a Toral de los Vados (14-4-72). Barrio de Nuestra Señora (29-6-73). Puente Villarente (19-10-72). Siempre en herbazales al borde de caminos. León (TUXEN/OBERDORFER).

Subcosmopolita. 
Lactuca tenerrima Pourret

Pantano del Porma (9-7-72), en calizas. Citas bibliográficas, no muy numerosas, en La Montaña.

Mediterránea-Atlántica. Ononidetalia striatae.

Cicerbita alpina (L.) Wallr.

Mulgedium alpinum Lessing

Peña Cuiña, Ancares (25-7-70). Cofiñal (13-8-72). Sustrato silíceo. Se le ha citado de la zona montañosa nororiental.

Orófila subalpina. Europea.

\section{Lampsana communis $\mathrm{L}$.}

Barrio de Nuestra Señora (29-6-72), en borde de carretera. Muy citado en toda la provincia.

Eurosiberiana.

Crepis foetida $\mathrm{L}$.

Sena de Luna (30-6-71). Villafranca del Bierzo (LANGE).

Europea.

Crepis paludosa (L.) Moench

Villafeliz de Babia (1-7-71). Cofiñal (13-8-72). Puerto de San Glorio (6-8-72), en prados encharcados. Nocedo (BORJA). San Emiliano (MAYOR y col.). Vegamián (I.N.I.A.).

Eurosiberiana.

Crepis mollis (Jacq.) Ascherson

Crepis succisifolia Tausch

Valporquero (19-8-72).

Orófila subalpina europea.

Crepis vesicaria $\mathrm{L}$.

subsp. haenseleri (Boiss.) P. D. Sell

Crepis taraxacifolia Thuill.

Luyego (1-5-73). Puente de Domingo Flórez (23-4-72). Virgen del Camino (12-5-73). Pola de Gordón (31-5-73). Orillas de caminos.

Submediterránea.

Crepis capillaris (L.) Wallr.

Crepis virens $\mathrm{L}$.

Sena de Luna (30-6-71), en prados de siega. Castro del Condado (11-7-70), en brezales. Vegacervera (11-7-70), en prados. Muy citada en La Montaña.

Europea.

Hieracium murorum $\mathrm{L}$.

Catoute (26-7-73). Las Médulas y Congosto (LANGE). La Guiana y San Pedro de Montes (POURRET). Convento de Arbas (BOURGEAU). Puebla de Lillo (RIVAS MARTfNEZ). Vegamián (I.N.I.A.). 
Hieracium pellitum Fries

Valverde de Curueño (18-6-72), sobre sustrato calcáreo.

Orófila alpina.

\section{ALISMATACEAE}

Alisma repens Cav.

Ardoncino (14-5-72), en charcas.

Mediterránea-Atlántica. Isoeto-Nanojuncetea.

\section{LILIACEAE}

Simethis planifolia G. G.

Anthericum planifolium L. A. bicolor Desf.

Benllera (20-6-70). Sorribos de Alba (6-7-70), siempre en brezales. La Guiana (POURRET).

Mediterránea-Atlántica.

Anthericum liliago $\mathrm{L}$.

Valporquero (19-8-72), cerca de un hayedo. Riaño (LAfNZ).

Submediterránea-Atlántica.

Gagea bohemica Roem./Sch.

Tabladillo (18-3-73), en campos de cultivo.

Mediterráneo occidental.

Gagea lutea Ker.

Santa Colomba de Somoza (18-3-73). León (QUER). La subsp. burnatil, en distintas zonas de la Cantábrica (LAfNZ).

Mediterránea.

Tulipa australis Link

$T$. alpestris Jord./F.

Crémenes (16-6-72). Valporquero (19-8-72). Siempre sobre sustrato calcáreo. Entre Portilla y Llánaves de la Reina (LAfNZ).

Mediterránea y centroeuropea. Ononidetalia.

Fritillarla messanensis Rof.

La Bañeza (6-5-73), en un encinar.

Ibérica. Rosmarinetalia.

Allium fallax Roem.

La Uña (13-8-72), en calizas. Citado también en el nordeste de la provincia. Orofila alpina. En Ononidetalia striatae.

Allium schoenoprasum $\mathrm{L}$.

Puerto de San Glorio (6-8-72). Coriscao (LOSA).

Orófila alpina. 


\section{Allium victorialis $\mathrm{L}$.}

Catoute (26-7-73), en zonas silíceas muy húmedas. Puebla de Lillo (LOSA/MONTSERRAT). Entre Sajambre y Valdeón (LAINZ).

Boreal ártico-alpina.

\section{Allium sphaerocephalum $\mathrm{L}$.}

La Uña (13-8-72). Utrero (9-7-72). Sobre calizas en ambos casos. Citas abundantes en el nordeste de la provincia.

Mediterránea. Brometalia.

\section{Scilla verna Huds.}

Herreros de Jamuz (6-5-73). Pinar de Puebla de Lillo (17-6-73). Citas bibliográficas en la zona montañosa nororiental, no muy numerosas.

Mediterránea-Atlántica.

\section{Paris quadrifolia L.}

Puebla de Lillo (19-7-70), en un hayedo. Citas bibliográficas muy abundantes en La Montaña.

Orófila alpina. Fagion.

Polygonatum officinale All.

P. odoratum (Mill.) P. F.

Carretera de Villamanín a Aralla (13-6-70). Valporquero (22-7-73). Bierzo (ROTHMALER).

Mediterránea-Atlántica.

Asparagus acutifolius $\mathrm{L}$.

Herreros de Jamuz (6-5-73), en cultivos de secano. Citas bibliográficas en el centro y sur de la provincia.

Circunmediterránea.

\section{AMARYLLIDACEAE}

Narcissus asturiensis (Jordan) Pugsley

Minas de Talco, Puebla de Lillo (10-5-71). Puerto de la Magdalena, Valverde, Vegarada, San Isidro (RIVAS-MARTÍNEZ, IZCO y COSTA).

Endemismo regional.

\section{DIOSCOREACEAE}

\section{Tamus communis L.}

Burbia (26-7-70). Puente de Domingo Flórez (23-4-72). Villafranca del Bierzo (LANGE). San Pedro de Montes (POURRET). Garganta del Cares (LASCOMBES). Mediterránea-Atlántica.

\section{IRIDACEAE}

\section{Crocus vernus All.}

Santa Colomba de Somoza (18-3-73). Sustrato pizarroso.

Sur de Europa. 


\section{Gladiolus illyricus Koch.}

Pantano del Porma (9-7-72), sobre sustrato calcáreo.

Circunmediterránea.

\section{JUNCACEAE}

Luzula forsteri DC.

Parandones (14-4-72). Lodares (29-6-72). Citas bibliográficas en La Montaña y E1 Bierzo.

Mediterránea-Atlántica. Fagion.

Luzula silvatica Gaud.

Pinar de Puebla de Lillo (17-6-73). Citas bibliográficas en el nordeste provincial, no muy numerosas.

Paleotemplada. Fagion.

\section{GRAMINEAE}

Phalaris arundinacea $L$.

Boñar (13-7-74), en borde de acequia. Citas bibliográficas esporádicas en la provincia. Circumboreal. Phragmition.

Phalaris coerulescens Desf.

Lodares (29-6-72).

Mediterránea.

Phleum phleoides (L.) Karst.

Villafranca del Bierzo (14-4-74).

Eurosiberiana.

Alopecurus myosuroides Hudsosn

A. agrestis $\mathrm{L}$.

Zotes del Páramo (14-5-72). San Miguel del Camino (12-5-73). En campos de cultivo en ambos casos. Una cita en León de LANGE.

Circumboreal.

\section{Phragmites communis Trin.}

Villafeliz de Babia (1-7-71), en borde de acequia. Citas bibliográficas diseminadas por toda la provincia.

Cosmopolita. Phragmition.

Agrostis alpina Scop.

Catoute (26-7-73); sustrato silíceo. Citas muy abundantes en los puertos de montaña. Orófila europea.

Agrostis castellana Boiss./Reuter

Vegamián (27-7-70), en borde de pastizal. Nocedo (BORJA). Villafranca del Bierzo (LANGE). Vegamián y Colle (I.N.I.A.). Puerto de Manzanal (TUXEN/OBERDORFER).

Mediterránea. Cisto-Lavanduletea. 
Agrostis salmantica (Lag.) Kunth.

Santa María del Condado (11-7-70), en brezales.

Ibérica.

Agrostis truncatula Parl.

Puebla de Lillo (1-8-71), en brezales. Catoute (26-7-73). Citas bibliográficas abundantes en la zona montañosa del norte de la provincia.

Ibero-Mediterránea.

Stipa lagascae R. Sch.

Herreros de Jamuz (28-5-73), en bordes de caminos.

Ibérica.

Piptatherum paradoxum P. B.

Vega de Gordón (31-5-73), sobre sustrato calcáreo. Nocedo (BORJA). Vegamián (I.N.I.A.).

Mediterránea.

Milium vernale $M$. B.

Brañuelo (23-5-71), en suelos ácidos.

Eurasia y Mediterráneo.

Periballia hispanica Trin.

De Villafranca a Toral de los Vados (14-4-72). Villafranca del Bierzo (LANGE). San Pedro de Montes (POURRET).

Ibérica.

Airopsis minuta Desv.

Marne (19-4-69), en pastizales. Citada en la vertiente berciana del puerto de Manzanal.

Circunmediterránea.

Aira multiculmis Dum.

Luyego (1-5-73), en cultivos abandonados.

Subcosmopolita.

Deschampsia flexuosa (L.) Trin.

Peña Cuiña, Ancares (25-7-70). Catoute (26-7-73). En ambos casos la var. brachyphylla Gay. Citas bibliográficas muy abundantes en La Montaña.

Mediterránea-Atlántica.

Deschampsia media R. S.

Vegacervera (11-7-70). Cofiñal (13-8-72). En pastizales. Numerosas citas bibliográficas en La Montaña.

Submediterránea. Deschampsion medieae.

Deschampsia refracta R. S.

Vegas del Condado (3-7-69). León (LAGASCA).

Molinietalia. 


\section{Avena sterilis $\mathrm{L}$.}

Sena de Luna (1-7-71), en borde de carretera. Arrabalde (I.N.I.A.).

Mediterránea.

Gaudinia fragilis P. B.

Cistierna (12-6-69). Sena de Luna (30-6-71). En prados de siega. Cerca de la ciudad de León (LAGASCA).

Iberomediterránea. Molinio-Arrhenatheretea.

Sieglingia decumbens (L.) Bernh.

Dantonia decumbens (L.) Lam.

Villargusán (26-6-69). Benllera (28-6-70). Teleno (8-8-71), en lugares muy húmedos. Villafranca del Bierzo (LANGE). Nocedo (BORJA). Vegamián (I.N.I.A.).

Europea.

Koeleria phleoides Pers.

Astorga (14-5-72), en cultivos. Citada en La Montaña y El Bierzo.

Mediterránea. Hordeion.

Koeleria vallesiana (Sut.) Gaud.

subsp. vallesiana

var. abreviata

Cistierna (12-6-69). Piedrafita de Babia (13-6-71). Pantano del Porma (16-7-71). Valporquero (19-8-72). En calizas. Citas bibliográficas numerosas en La Montaña. Mediterránea.

uPoa ligulata Boiss.

Lugán (5-6-69). Crémenes (12-6-72). Valporquero (19-8-72). Sobre sustrato calcáreo. Citas bibliográficas en la mitad norte de la provincia.

Ibérica. Ononidion striatae.

Melica ciliata L.

Puebla de Lillo (1-8-71). Puente de Domingo Flórez (19-5-72). Citas bibliográficas numerosas en La Montaña y El Bierzo.

Mediterránea. Asplenietea y Thlaspeetea.

\section{Melica uniflora Retz}

Valporquero (19-8-72), en hayedos sobre sustrato calcáreo. Citas bibliográficas en La Montaña y El Bierzo.

Mediterránea-Atlántica. De Fagion.

Dactylis glomerata L.

subsp. aschersoniana (Graebn.) P. F.

Valporquero (19-8-72).

Paleotemplada.

Cynosurus elegans Desf.

Camposagrado (13-6-69). Crémenes (16-6-72). Vega de Gordón (31-5-73). Villanueva del Bierzo (19-5-72). Siempre en pastizal. Nocedo (BORJA).

Europa mediterránea. 
Wangenheimia lima Trin.

Cynosurus lima Loefl.

Lago de Carucedo (25-5-74). San Esteban del Molar (I.N.I.A.).

España. Rosmarinetalia y Thero-Brachypodietalia.

Vulpia ciliata Link

Ribaseca (28-5-73), en cultivo de cereales en barbecho. Montes de León (LANGE). Puerto de Manzanal (TUXEN/OBERDORFER).

Sur de Europa, Asia occidental y África septentrional.

Festuca ovina L.

subsp. laevis Hack.

Peña Cuiña, Ancares (25-7-70). Valporquero (19-8-72). Puebla de Lillo, Cebolledo (17-6-73).

Festuca pumila Chaix.

subsp. eskia (Ram.) Lit.

Puerto de San Glorio (6-8-72). Citas bibliográficas en la zona montañosa nororiental, poco numerosas.

Pirineos y Cordillera Cantábrica. Caricetalia curvulae.

Fesiuca rubra L.

subsp. rivularis Boiss.

Puerto de San Glorio (6-8-72), en bordes de arroyos.

Bromus maximus Desf.

Puente Castro (11-6-72). Barrio de Nuestra Señora (19-8-72). Lorenzana (10-6-73). Citas bibliográficas en casi toda la provincia.

Paleo-subtropical.

Bromus mollis L.

Ponferrada (12-5-73).

Subcosmopolita.

Bromus rubens $\mathrm{L}$.

Puente Castro (28-6-70).

Paleo-subtropical.

Hordeum murinum L.

Virgen del Camino (12-5-73). Ribaseca (28-5-73). Citas bibliográficas numerosas en toda la provincia.

Circumboreal. De Hordeion.

Elymus caput-medusae L.

Vegas del Condado (22-6-69), en terrenos muy secos y arcillosos. Citas bibliográficas en la Meseta leonesa; poco numerosas.

Mediterránea. 
Agropyrum intermedium (Host.) Beauv.

Sena de Luna (30-6-71), en bordes de prados.

Europa mediterránea.

Brachypodium pinnatum P. Beauv.

Vegacervera (11-7-70). Palazuelo de Boñar (29-6-73), en desmontes y colinas. Citas bibliográficas en el centro y oriente del tramo leonés de la Cordillera Cantábrica. Mediterránea. De Brometalia.

\section{Brachypodium silvaticum R. S.}

Puebla de Lillo (1-8-71). Valporquero (19-8-72). Substrato calcáreo. Citas bibliográficas en El Bierzo y zona montañosa nororiental.

Mediterránea. En Fagion y Populetalia albae.

Lolium multiflorum Lam.

Vegas del Condado (1-7-69). La Bañeza (LANGE). Puebla de Cilleros (I.N.I.A.). Mediterránea-Atlántica.

Lolium perenne $\mathrm{L}$.

Villargusán (29-6-69). Vegas del Condado (1-7-69). Sena de Luna (30-6-71). Villasabariego (11-6-72). Citas bibliográficas en La Montaña y El Bierzo.

Circumboreal. Lolio-Plantaginetum.

\section{ARACEAE}

\section{Arum maculatum $\mathrm{L}$.}

Villafranca del Bierzo (14-4-72). Crémenes (16-6-72). San Pedro de Montes (POURRET). Riaño (LOSA). Burón, Oseja de Sajambre y Riaño (I.N.I.A.).

Europea. Fagion.

\section{CYPERACEAE}

Elyna myosuroides (Vill.) Fritsch.

Puebla de Lillo, Cebolledo (11-7-73), a 1.600 m.s.n.m. Base de Torrecerredo, La Padiorna, sobre la Vega de Liordes (LAfNZ).

Holoártica-alpina. Elyno-Seslerietea.

\section{Carex verna Chaix.}

C. praecox Jacq. C. caryophyllea Fiori.

Lago de Carucedo (23-4-72). San Glorio, Tarna y Coriscao (LOSA/MONTSERRAT). Euroasiática.

\section{Carex acutiformis Ehrh.}

C. paludosa Good.

Lorenzana (11-6-73). Crémenes (13-6-73). Pinar de Lillo (17-6-73). En charcas. Navatejera, Villaquilambre (LAfNZ).

Euroasiática. 
Carex echinata Murr.

C. stellulata Good.

Puerto de San Glorio (6-8-72). Torrestío (10-6-73). Teleno (8-8-71). Lodares (26-6-72).

En zonas muy húmedas. Citadas en La Montaña leonesa.

Circumboreal. Caricion fuscae.

Carex glauca Murr.

Puente de Domingo Flórez (23-4-72). Aviados (4-6-72). Lodares (29-6-72). Citas bibliográficas en La Montaña; no muy numerosas.

Europea. De Molinio-Holoschoenion.

Cârex oederi Retz.

subsp. aederi

Crémenes (11-6-72). Lodares (29-6-72).

Circumboreal.

Carex paniculata $L$.

Huerga de Garaballes (23-5-71). Armellada (12-5-72). Villadepalos (15-4-72). La Bañeza (LANGE).

Mediterránea-Atlántica. De Magno-Caricion.

Carex pilulifera $\mathrm{L}$.

Catoute (26-7-73). Laciana Alta (LAfNZ). Puerto de Pajares (TUXEN/OBERDORFER).

Europa atlántica.

Carex pulicaris L.

Puerto Ventana (2-7-69). Busdongo (LAGASCA).

Eurosiberiana.

Carex rostrata With.

Puerto de San Glorio (6-8-72). Lago Ausente, Puebla de Lillo (ALLORGE).

Circumboreal. Molinio-Juncetea y Phragmition.

Carex sempervirens Vill.

Puerto de San Isidro, Cebolledo (1-7-73). Abundantes citas bibliográficas en la Montaña leonesa.

Orófila alpina. Asplenietea rupestris.

\section{ORCHIDACEAE}

Epipactis latifolia All.

Valporquero (19-8-72), en sustrato calcáreo.

Paleotemplada.

Coeloglossum viride (L.) Hartmann

Cofiñal (13-8-72), en prados al borde del río. San Pedro de Montes (POURRET). Circumboreal. 


\section{Orchis bifolia $\mathrm{L}$.}

Platanthera bifolia Rich.

Puente Orugo (30-6-71), en prados de siega.

Euroasiática.

Orchis conopea (L.) R. Br.

Camposolillo (29-6-73). Citas bibliográficas en La Montaña; no muy numerosas.

Eurosiberiana.

\section{Orchis latifolia $\mathrm{L}$.}

Puebla de Lillo (16-6-73), en prados húmedos.

Europea. Molinietalia.

Orchis longibracteata Biv.

Aceras longibracteata Reichenb.

Loroglossum longibracteatum (Biv.) Moris

Barlia longibracteata Palat.

Sena de Luna (1-7-71).

Mediterránea.

\section{Orchis pallens $\mathrm{L}$.}

Santa Lucía (11-6-73), sobre sustrato calcáreo. Puerto de Pandetrave y Ubiña (LAINRZ).

Orófila europea.

Orchis pyramidalis $\mathrm{L}$.

Anacamptis pyramidalis Rich.

Valporquero (22-7-73), sobre sustrato calcáreo. Torrestío (DUPONT, P./S.).

Mediterránea europea.

Ophrys scolopax Cav.

Peña Rubia (19-5-73). Pola de Gordón (31-5-73).

Mediterráneo occidental.

Aceras antropophora $\mathrm{R}$. Br.

Crémenés (12-6-72); Miñera de Luna (1-7-71). En ambos casos en suelos poco profundos sobre sustrato calcáreo. Mirantes de Luna (RIVAS MARTINEZ y col.). Mediterránea-Atlántica.

\section{BIBLIOGRAFIA}

Allorge, P. 1927. Sur la végétation des bruyères a sphaigne de la Galice. Compt. Rend. Ac. Sc., París.

Allorge, V. / P. 1941. Plantes rares ou interessantes du N. W. de l'Espagne. Bull Soc. Bot. France, $88: 226-254$.

Amaral Franco, J. 1968. Distribuçao de zimbros e pomoideas na Península Ibérica. Collect. Bot., 7(1): 449-481, Barcelona.

Andrés, J. 1969. Algunas plantas del Ayuntamiento de Puebla de Lillo (León). Rev. Fac. Cienc. Oviedo. N. S., Vol. XI (2):125-129. 
Andrés, J. / Carbó, R. 1969. Algunas plantas del partido farmacéutico de Vegas del Condado (León). Monitor de la Farmacia. N. ${ }^{\circ}$ 19. 64:371-374.

Bellot, F. 1951. Sipnosis de la vegetación de Galicia. Anal. Inst. Bot. A. J. Cavanilles. X:389-444.

Bellot, F. 1966. La vegetación de Galicia. Anal. Inst. Bot. A. J. Cavanilles. XXIV: 1-306.

Benito Cebrián, N. 1948. Brezales y brezos. Inst. Forest. Inv. y Exper. Vol. XXXIX.

Bernis, F. 1949. Sobre los tipos que presenta Agrostis tenuis Sibth. en Maragatería (León). Anal. Soc. Esp. Hist. Nat. (2.a), 47:145-146. Madrid.

Bernis, F. 1953. Revisión del género Armeria Willd., con especial referencia a los grupos ibéricos. Anal. Inst. Bot. A. J. Cavanilles. XII(2):77-252.

Borja Carbonell, J. 1952. Datos para la flora cantábrica. Plantas de Nocedo (León). Anal. Inst. Bot. A. J. Cavanilles. XI(1):419-436.

Borja Carbonell, J. 1952 b. Una visita a la localidades clásicas del Geranium subargenteum Lge., Onobrychis reuteri Leresche y a los brezales de Mabe. Anal. Inst. Bot. A. J. Cavanilles. X(2):399-412.

Braun-Blanquet, J. 1936. La lande á Romarin et Bruyère (Rosmarino-Ericion) en Languedoc. S.I.G.M.A. Comm. 48.

Braun-Blanquet, J. y col. 1940. Prodrome des groupements végétaux. Fasc. 7. (Clase Cisto-Lavanduletea) Montpellier.

Braun-Blanquet, J. / Pinto da Silva, A. / Rozeira, A. \& Fontes, F. 1952. Résultats de deux excursions géobotaniques à travers le Portugal septentrional et moyen, I. Agron. Lusit. Vol. 14. Sacavem.

Braun-Blanquet, J./ Pinto da Silva, A. / Rozeira, A. 1956. Résultats de deux excursions géobotaniques à travers le Portugal septentrional et moyen, II. Agron. Lusit., 18(3). Sacavem.

Braun-Blanquet, J. / Pinto Da Silva, A. / Rozeira, A. 1964. Résultats de trois excursions géobotaniques à travers le Portugal septentrional et moyen, III. Agron. Lusit., XXIII(4).

Caballero, A. 1941. Ilustraciones de la flora endémica española. Anal. Jard. Bot. Madrid, II : 266-348.

Caballero, A. 1942. Ilustraciones de la flora endémica española. Anal. Jard. Bot. Madrid, III : 328-381.

Caballero, A. 1945. Ilustraciones de la flora endémica española. Anal. Jard. Bot. Madrid, VI(2): 549-591.

Caballero, A. 1947. Ilustraciones de la flora endémica española. Anal. Jard. Bot. Madrid, VIII : 523-579.

Camará Niño, F. 1955. Plantas de montañas españolas. Anal. Est. Exper. Aula Dei, III : 267-352. Zaragoza.

Carbó, R. / Mayor, M. / Andrés, J. / Losa, J. M. 1972. Aportaciones al catálogo florístico de la provincia de León. Anal. Fac. Vet. León, XVIII(1):225-352.

Castroviejo, S. 1973. El área suroccidental de los brezales gallegos. Anal. Inst. Bot. A. J. Cavanilles, XXX:197-213.

Colmerro, M. 1872. Genisteas y Anthyllideas de España y Portugal. Anal. Soc. Esp. Hist. Nat. $\left(10^{\mathrm{a}}\right): 1-289$.

Colmeiro, M. 1874. Plantas crasas de España y Portugal, o sea, enumeración de las crasuláceas, ficoideas y cactáceas que crecen espontáneamente en la Península o se han naturalizado en ella. Anal. Soc. Esp. Hist. Nat. (1. $\left.{ }^{\mathrm{a}}\right)$, $3: 267-299$. Madrid.

Colmeiro, M. 1885. Enumeración de las plantas de la Península hispanolusitana. Edit. Imprenta de Vda. e Hijas de Fuentenebro. Madrid.

Comte, P. 1937. La série cambrienne et silurienne du León. Espagne. Sci. París, T. 204: 604-606.

Coste, H. 1937. Flore descriptive et illustrée de la France. Libraire des Sciences et des Arts. París. T.: I, II, III.

Dresser, D. W. 1959. Notes on the prealpine flore of the Picos de Europa, Spain, I. Not. R. Bot. Gard. Edimb., XXIII : 25-46.

Dupont, P. 1953. Contribution a la flore du Nord-Ouest de l'Espagne, I. Bull. Soc. Hist. Nat. Toulouse, $88: 120-132$. 
Drspont, P./S. 1956. Aditions a la flore du nord-ouest de l'Espagne, I. Bull. Soc. Hist. Nat. Toulouse, $91: 313-334$.

Font QueR, P. 1948. Acerca de algunas plantas raras, críticas o nuevas. Collect. Bot. Barcelona, I(3): 211-314.

Gandoger, M. 1895. Voyage botanique aux Picos de Europa et dans les provinces du Nord-Ouest de l'Espagne. Bull. Soc. Bot. France, 42:1-49.

Gandoger, M. 1898. Notes sur la flore espagnole, III. Mon sixiéme voyage dans la Pen. Iberique. Bull. Soc. Bot. France, 45:591-595.

Giménez, M. A.,/ LosA, J. M. 1974. La estructura del bosque de Quercus rotundifolia Lam. en la comarca del Bierzo (León). Trab. Comp. de Biolog., 4: 41-102.

GimÉNEZ, M. A. 1976. Estudio de comunidades vegetales naturales en la comarca del Bierzo. Servicio de Mecanización de la Universidad de Santiago de Compostela.

Guinea, E. 1946. De mi primer viaje botánico a los Picos de Europa. Anal. Inst. Bot. A. J. Cavanilles, VII : 335-356.

Guitian Ojea, F. 1964. Técnicas de análisis de suelos. C.S.I.C. Monografías. Madrid. Pp.: 50-53.

Heywood, V. H. 1953. A revision of the Spanish species of Tanacetum L. Subsect. Leucanthemopsis Giroux. Anal. Inst. Bot. A. J. Cavanilles, XI:314-378.

I.N.I.A. (Instituto Nacional de Investigaciones Agrarias), 1973. Mapa provincial de suelos (León). Departamento Nacional de Ecología. Madrid.

IUlivert, M. 1967. La ventana del río Monasterio y la terminación meridional del manto del Ponga. Trabajos de Geología. Universidad de Oviedo. Pp.: 59-76.

LaCaIta, C. 1928. Novitia quedam et notabilia hispanica. Cavanillesia, I:6-15; III: $20-47$.

Lacaita, C. 1929. Observation sur la flore des Picos de Europa. Bull. Soc. Bot. Gèneve. 2. ${ }^{\mathrm{a}}$ serie. $21: 135-142$. Genéve.

Lacaita, C. 1930. Rectification a la flore des Picos de Europa. Bull. Soc. Bot. Genève. $2{ }^{a}$ serie, $21: 263$.

Ladero Alvarez, M. 1970. Contribución al estudio de la flora y vegetación de las comarcas de La Jara, Serranía de Ibor y Guadalupe-Villuercas, en la Oretana. Madrid. Tesis Doctoral.

Laínz, M. 1954. Contribución al catálogo de la flora montañesa. Collect. Bot., 4(2). Barcelona.

Laf́nz, M. 1955. Adiciones al catálogo de la flora montañesa. "Altamira". Revista del Centro de Estudios montañeses: 3-13. Santander.

Laínz, M. 1956. Aportaciones al conocimiento de la flora montañesa. I. Collect. Bot., 5(1): 147-158. Barcelona.

Laínz, M. / Col. 1957. Aportaciones al conocimiento de la flora cántabro-astur., II. Collect. Bot., 5(2):429-460. Barcelona.

Laínz, M. / Col. 1959. Aportaciones al conocimiento de la flora cántabro-astur., III. Collect. Bot., 5(3):671-696. Barcelona.

Laínz, M. / Col. 1960. Aportaciones al conocimiento de la flora cántabro-astur. IV. Bol. Inst. Est. Ast. (C), 1:3-42. Oviedo.

LaínZ, M. / Col. 1961. Aportaciones al conocimiento de la flora cántabro-astur., V. Bol. Inst. Est. Ast. (C), 3:48-186. Oviedo.

Laínz, M. / Col. 1962. Aportaciones al conocimiento de la flora cántabro-astur. VI. Bol. Inst. Est. Ast. (C), 5:3-43.

LAínZ, M. / Col 1963. Aportaciones al conocimiento de la flora cántabro-astur. VII. Bol. Inst. Est. Ast. (C), 7:35-81. Oviedo.

LaínZ, M. / Col. 1964. Aportaciones al conocimiento de la flora cántabro-astur. VIII. Bol. Inst. Est. Ast. (C), 10:173-218. Oviedo.

LAínZ, M. / Col. 1970. Aportaciones al conocimiento de la flora cántabro-astur. IX. Bol. Inst. Est. Ast. (C.), 15:3-45. Oviedo.

LaínZ, M. / Col. 1973. Aportaciones al conocimiento de la flora cántabro-astur. X. Bol. Inst. Est. Ast. (C.), 16:159-206. Oviedo. 
Lascombes, G. 1944. La végétation des Picos de Europa. Les paysages forestiers. Bull. Soc. Hist. Nat., Toulouse. 79:339-358.

LAWAlREe, A. 1956. Quelques fougéres d'Espagne. Ergebnisse de 10, I.P.E. dur Spanien, 1953. Zurich.

Lautensach, H. 1967. Geografía de España y Portugal. Edit. Vicens Vives. Barcelona.

Leresche, L. Levier, E. 1880. Deux excursions botaniques dans le Nord de l'Espagne et le Portugal. Imprimerie Georges Bridel. Lausanne.

Leroy, E. / Laínz, M. 1954. Contribución al catálogo de la flora palentina. Collect. Bot., 4(1): 81-123. Barcelona.

Litardiere, R. de 1911. Contribution a l'etude de la flore pteridologique de la Peninsule Iberique. Bull. Acad. Int. Geogr. Bot. :12-30.

LitARDIERE, R. de 1951. Sur la repartition en Espagne du groupe du Fetuca ovina subsp laevis Hacq. (var. gallica et var. marginata Hack.). Anal. Jard. Bot. Madrid, X (2):291-300.

Litardiere, R. de 1954. Contribution a l'etude du Festuca burnatii St.-Yves. Collect. Bot. IV(1): 179-181. Barcelona.

Losa España, T. M. 1941. Plantas de los alrededores de Riaño. Anal. Jard. Bot. Madrid, III : 172-187.

Losa España, T. M. 1946. Algo sobre las especies españolas del género Euphorbia L. Anal. Jard. Bot. Madrid, VII : 357-432.

Losa España, T. M. 1949. Contribución al estudio de la flora y vegetación de la provincia de Zamora. C.S.I.C. Inst. A. I. Cavanilles. Sección de Barcelona.

Losa España, T. M. 1954. Resumen de un estudio comparativo de la flora de los Pirineos franco-españoles y los montes cántabro-leoneses. Anal. Inst. Bot. A. J. Cavanilles, XIII:233-268.

Losa EsPaÑa, T. M. 1957. Catálogo de las plantas que se encuentran en los montes palentino-leoneses. Anal. Inst. Bot. A. J. Cavanilles, XV:243-376.

Losa España, T. M. 1958. El género Ononis L. y las Ononis españolas. Anal. Inst. Bot. A. J. Cavanilles, XVI:227-238.

Losa España, T. M. 1962. Los plántagos españoles. Anal. Inst. Bot. A. J. Cavanilles, $\mathrm{XX}: 5-50$.

Losa España, T. M. 1963. Especies españolas del género Chaenorrhinum Lge. Anal. Inst. Bot. A. J. Cavanilles, XXI: 543-572.

Losa, T. M. / Montserrat, P. 1952. Aportación al estudio de la flora de los montes cántabro-leoneses. Anal. Inst. Bot. A. J. Cavanilles, X(2):413-509.

Losa, T. M. / Montserrat, P. 1953. Nueva aportación al estudio de la flora de los montes cántabro-leoneses. Anal. Inst. Bot. A. J. Cavanilles, XI(2): 385-462.

Lotze, F. 1956. Das Prä kambrium Spaniens. N. Jb. f. Geol. Palaont. Min., Vol. 8: 377-380.

LotZe, F. 1957. Zum Alter nordwestspanischer Quartzit-Sandstem-Folgen. $N . J b$. Geol. Paläont. Mh., 10:464-471. Stuttgart.

Lucena Conde, F. / Col. 1961. La fertilidad química de los suelos en la provincia de León. Centro de Edafol. y Biol. Aplicada de Salamanca-León (C.S.I.C.) :15-20. Publicaciones de la Excma. Diputación Provincial de León.

Martín Bolaños, M. / Guinea, E. 1949. Jarales y jaras (Cistografía hispánica). Inst. Forest. Inv. y Exp., Año XX, N. ${ }^{\circ} 49$.

Martínez García, G. 1973. Estudio de la flora y vegetación de las comarcas comprendidas entre Trubia (Asturias) y San Emiliano (León). Tesis Doctoral.

Martínez, G. / Mayor, M. \& Navarro, F. y Df́az, T. E. 1974. Estudio fitosociológico y fitotopográfico de la vertiente septentrional y meridional del Puerto de Ventana. Rev. Fac. Cienc. XV(1):55-109. Oviedo.

Matte, Ph. 1964. Remarques preliminaires sur l'allure des plis hercy niens en Galice orientale. C. R. Acad. Sc. 259:1.981-84. París.

Matte, Ph. 1968. La structure de la virgation hercynienne de Galice (Espagne). Geol. Alpine, 44:1-127. Grenoble. 
Mayor, M./Andrés, J. 1969. Juncus striatus Schousb. en Vegas del Condado (León). Rev. Fac. Cienc. XI(2): 131-133. Oviedo.

Mayor, M. / ANDRÉs, J. 1968. Festulolium loliaceum (Huds.) P. Fourn. en Villargusán (León). Trab. Depart. Bot. y Fisiol. Veget. I(3): 149-152. Facultad de Ciencias. Madrid.

Mayor, M. / Andrés, J./Martínez, G. 1970. Comportamiento fitosociológico de Pulsatilla rubra subsp. hispánica en algunas localidades de la Península Ibérica. Rev. Fac. Cienc. XI(2):297-304. Oviedo.

Mayor, M. / Andrés, J. / Martínez, G. / Navarro, F. / Díaz, T. E. 1973. Estudio de los pastizales de diente y siega en algunas localidades de la Cordillera Cantábrica con especial atención al comportamiento ecológico de Festuca hystrix Boiss. Rev. Fac. Cienc. 14(2): 161-171. Oviedo.

Montserrat Recoder, P. 1963. El género Luzula en España. Anal. Inst. Bot. A. I. Cavanilles. XXI(2): 407-542.

Navarro Andrés, F. 1974. Estudio de la Flora y Vegetación de la Sierra de Aramo y sus estribaciones (Asturias). Tesis Doctoral.

NAVArro Andrés, F. 1974. La vegetación de la Sierra del Aramo y sus estribaciones (Asturias). Rev. Fac. Cienc. XV(1):111-243. Oviedo.

PaU, C. 1928. Notas de mi herbario. Cavanillesia. I(4-6):60-67. Barcelona.

Paunero, E. 1946. Las especies españolas del género Agrostis. Anal. Inst. Bot. A. J. Cavanilles. VII : 561-644.Madrid.

Paunero, E. 1947. Revisión de las especies españolas del género Phalaris. Anal. Inst. Bot. A. J. Cavanilles. VIII:475-522. Madrid.

Paunero, E. 1948-49. Las especies españolas del género Trisetaria. Anal. Inst. Bot. A. I. Cavanilles. XI: 503-582. Madrid.

Paunero, E. 1951. Las especies españolas del género Alopecurus. Anal. Inst. Bot. A. J. Cavanilles. $\mathrm{X}(2): 301-346$. Madrid.

Paunero, E. 1952. Las agrostideas españolas. Anal. Inst. Bot. A. J. Cavanilles. XI(1): 319-418. Madrid.

Paunero, E. 1953. Las especies españolas del género Anthoxanthum L. Anal. Inst. Bot. A. J. Cavanilles. XII(1): 401-442. Madrid.

Paunero, E. 1954. Las aveneas españolas. Anal. Inst. Bot. A. J. Cavanilles. XIII(1): 149-230.Madrid.

Paunero, E. 1955. Las aveneas españolas, II. Anal. Inst. Bot. A. J. Cavanilles. XIV : 187-252. Madrid.

Rivas Goday, S. 1947. Los brezales de España. Bol. Cons. Gen. Col. Farmac. España. Madrid.

Rivas Goday, S. 1949. Proyecto de nuevas alianzas de la clase Cisto-Lavanduletea. Bol. R. Soc. Esp. Hist. Nat. T. Extr. : 19-46.

Rivas Goday, S. 1957. Nuevos órdenes y alianzas de Helianthemetea annua. Anal. Inst. Bot. A. J. Cavanilles. XV:540-651. Madrid.

Rivas Goday, S./Col. 1959. Contribución al estudio de la Quercetea ilicis hispanica. Anal. Inst. Bot. A. J. Cavanilles. XVII(2):285-406. Madrid.

Rivas Goday, S. / Borja Carbonell, J. 1961. Estudio de la vegetación y flórula del macizo de Gúdar y Javalambre. Anal. Inst. Bot. A. I. Cavanilles. XIX:1550. Madrid.

Rivas Goday, S. / Rivas-Martínez, S. 1958. Una visita a la Laguna de Arvas (Leitariegos). Anal. Inst. Bot. A. J. Cavanilles. XVI:565-586. Madrid.

RivaS-MARTínez, S. 1964. Relación entre los suelos y la vegetación en la comarca de La Puebla de Lillo (León). Anal. Edaf. y Agrobiol. XXIII:323-333.

Rivas-Martínez, S. 1968. Los jarales de la Cordillera Central. Collect. Bot. Barcelona. VII : 1.033-1.082.

Rivas-Martínez, S. / Izco, J./Costa, M. (1971. Sobre la Flora y Vegetación del macizo de Peña Ubiña. Trab. Dep. Botánica y F. Veg. 3:47-123. Madrid.

Ron Álvarez, M. E. 1968. Comentarios a algunos areales de plantas de las obras de Walter, Meusel, Jäger y Weinert. Anal. Inst. Bot. A. J. Cavanilles. XXVI: 73-88. 
Rothmaler, W. 1935. Generum plantarum ibericarum revisio critica, III. Euphrasia L. Cavanillesia (1-5), $7: 15-28$.

Rothmaler, W. 1935. Plantae novae ad criticae Peninsula Ibericae. Cavanillesia 7(69): 111-121.

RothmaleR, W. 1954. Vegetationstudien in Nordwest-Spanien. Vegetatio, 5-6: 595-601.

SitTer, L. U. de 1962. The structure of the southern slope of the Cantabrian Mountains. Leidse Geol. Med. Vol. 26:255-264.

Sole SABARIS, L. 1949. Evolución morfológica comparada de tres macizos hercinianos españoles. C. R. Congr. Intern. Géogr. Lisbonne. T. II:674-691.

Suárez Menéndez, O. 1970. Estudio petrológico de los afloramientos plutónicos de la zona occidental astur-leonesa. Tesis Doctoral. Universidad de Salamanca.

Tutin, T. G. / Col. 1968. Flora Europaea. T.: I, II, III; Cambridge University Press.

TüXEN, R. / OBERDORFER, E. 1958. Eurosibirische Phanerogamen-Gesellschaften Spaniens. Geob. Inst. Rübel. 32:1-328.

Vicioso, C. 1945. Notas sobre la Flora española. Anal. Jard. Bot. Madrid, VI(2): 5-87.

Vicioso, C. 1951. Salicaceas de España. Inst. Forest. Inv. y Exper. Año XXII, N. ${ }^{\circ}$ 57. Madrid.

Vicioso, C. 1951. Tréboles españoles. Revisión del género Trifolium. Anal. Inst. Bot. Cavanilles. X(2): 347-398.

VIcioso, C. 1955. Genisteas españolas, II. Inst. Forest. Inv. y Exper. Año XXVI, 72. Madrid.

WAGNeR, R. H. 1962. Sur le terrain hoiller de Ciñera-Matallana (León, Espagne). C. R. Acad. Sci. Paris. V(254): 2.393-95.

Willkomm, M. / Lange, J. 1870. Prodromus Florae Hispanicae, I, II, III. Stuttgart.

Willкомм, M. 1893. Suplementtum Prodromi Florae Hispanicae. E. Schweizerbart. Stuttgart.

(Recibido el 2 de noviembre de 1977) 